\title{
Elko Spinor Fields and Massive Magnetic Like Monopoles
}

\author{
E. Capelas de Oliveira, W. A. Rodrigues Jr. and J. Vaz Jr. \\ Institute of Mathematics, Statistics and Scientific Computation \\ IMECC-UNICAMP \\ 13083-859 Campinas SP, Brazil \\ walrod@ime.unicamp.br, capelas@ime.unicamp.br, vaz@ime.unicamp.br
}

November 162013

\begin{abstract}
In this paper we recall that by construction Elko spinor fields of $\boldsymbol{\lambda}$ and $\boldsymbol{\rho}$ types satisfy a coupled system of first order partial differential equations (csfopde) that once interacted leads to Klein-Gordon equations for the $\boldsymbol{\lambda}$ and $\rho$ type fields. Since the csfopde is the basic one and since the KleinGordon equations for $\boldsymbol{\lambda}$ and $\boldsymbol{\rho}$ possess solutions that are not solutions of the csfopde for $\boldsymbol{\lambda}$ and $\boldsymbol{\rho}$ we infer that it is legitimate to attribute to those fields mass dimension $3 / 2$ (as is the case of Dirac spinor fields) and not mass dimension 1 as previously suggested in recent literature (see list of references). A proof of this fact is offered by deriving the csfopde for the $\boldsymbol{\lambda}$ and $\boldsymbol{\rho}$ from a Lagrangian where these fields have indeed mass dimension $3 / 2$. Taking seriously the view that Elko spinor fields due to its special properties given by their bilinear invariants may be the description of some kind of particles in the real world a question then arises: what is the physical meaning of these fields? Here we proposed that the fields $\boldsymbol{\lambda}$ and $\boldsymbol{\rho}$ serve the purpose of building the fields $\mathcal{K} \in \mathcal{C} \ell^{0}(M, \eta) \otimes \mathbb{R}_{1,3}^{0}$ and $\mathcal{M} \in \sec \mathcal{C} \ell^{0}(M, \eta) \otimes \mathbb{R}_{1,3}^{0}$ (see Eq.(38)). These fields are electrically neutral but carry magnetic like charges which permit them to couple to a $s u(2) \simeq \operatorname{spin}_{3,0} \subset \mathbb{R}_{3,0}^{0}$ valued potential $\mathcal{A} \in \sec \bigwedge^{1} T^{*} M \otimes \mathbb{R}_{3,0}^{0}$. If the field $\mathcal{A}$ is of short range the particles described by the $\mathcal{K}$ and $\mathcal{M}$ fields may be interacting and forming condensates of zero spin particles analogous to dark matter, in the sense that they do not couple with the electromagnetic field (generated by charged particles) and are thus invisible. Also, since according to our view the Elko spinor fields as well as the $\mathcal{K}$ and $\mathcal{M}$ fields are of mass dimension $3 / 2$ we show how to calculate the correct propagators for the $\mathcal{K}$ and $\mathcal{M}$ fields. We discuss also the main difference between Elko and Majorana spinor fields, which are kindred since both belong to class five in Lounesto classification of spinor fields. Most of our presentation uses the representation of spinor fields in the Clifford bundle formalism, which makes very clear the meaning of all calculations.
\end{abstract}




\section{Introduction}

Elko spinor fields have been introduced in [1, 2] as dual helicity eigenspinors of the charge conjugation operator satisfying Klein-Gordon equation and carrying according to the authors of [1, 2] mass dimension 1 instead of mass dimension $3 / 2$ carried by Dirac spinor fields. A considerable number of interesting papers have been published in the literature on these extraordinary objects in the past few years. In particular, according to the theory in [1, 2, the anticommutator of an elko spinor field with its conjugate momentum is nonlocal and it is claimed that the theory possess an axis of locality which implies also that the theory of elko spinor fields break Lorentz invariance. We shall discuss this issue in the Appendix B which according our view is an odd feature of the theory in [1, 2. We recall in section 2 that differently from the theory in [1, 2] where a second quantized elko spinor field satisfies a Klein-Gordon equation (instead of a Dirac equation) the classical elko spinor fields of $\boldsymbol{\lambda}$ and $\boldsymbol{\rho}$ types satisfy by their construction a csfopde that is Lorentz invariant. It is thus possible to construct (what we believe) is a more reasonable theory where those fields, as we will show play a key role. The csfopde once interacted leads to Klein-Gordon equations for the $\boldsymbol{\lambda}$ and $\boldsymbol{\rho}$ type fields. However, since the csfopde is the basic one and since the Klein-Gordon equations for $\boldsymbol{\lambda}$ and $\boldsymbol{\rho}$ possess solutions that are not solutions of the csfopde for $\lambda$ and $\rho$ we think that it is not necessary to get the field equations for $\boldsymbol{\lambda}$ and $\boldsymbol{\rho}$ from a Lagrangian where those fields have mass dimension 1 as in [1, 2]. Indeed, we claim that we can attribute mass dimension of $3 / 2$ for these fields as is the case of Dirac spinor fields. A proof of this fact is offered by deriving in Section 3 the csfopde for $\boldsymbol{\lambda}$ and $\boldsymbol{\rho}$ from a Lagrangian where these fields have mass dimension $3 / 2$. This, fact is to be contrasted with the quantum theory of these fields as presented in [1, 2, 5, 6, 28, 29, 34] (and references therein), namely that elko fields have mass dimension 1.

Taking seriously the view that elko spinor fields due to the special properties given by their bilinear invariants may be the description of some kind of particles in the real world a question then arises: what is the physical meaning of these fields?

In what follows we propose that the fields $\lambda$ and $\rho$ (the representatives in the Clifford bundle $\mathcal{C} \ell(M, \eta)$ of the covariant spinor fields $\boldsymbol{\lambda}$ and $\boldsymbol{\rho})$ serve the purpose of building Clifford valued multiform fields, i.e., $\mathcal{K} \in \mathcal{C} \ell^{0}(M, \eta) \otimes \mathbb{R}_{1,3}^{0}$ and $\mathcal{M} \in \sec \mathcal{C} \ell^{0}(M, \eta) \otimes \mathbb{R}_{1,3}^{0}$ (see Eq.(42)). These fields are electrically neutral but carry magnetic like charges which permit that they couple to a $s u(2) \simeq$ $\operatorname{spin}_{3,0} \subset \mathbb{R}_{1,3}^{0}$ valued potential $\mathcal{A} \in \sec \bigwedge^{1} T^{*} M \otimes \operatorname{spin}_{3,0}$. If the field $\mathcal{A}$ is of short range the particles described by the $\mathcal{K}$ and $\mathcal{M}$ may be interacting and forming a system of spin zero particles with zero magnetic like charge and eventually form condensates something analogous to dark matter, in the sense that they do not couple with the electromagnetic field and are thus invisible.

We observe that elko and Majorana fields are in class 5 of Lounesto classification 20] and although an elko spinor field does not satisfy the Dirac equation as correctly claimed in 1, 2, a Majorana spinor field $\psi_{\mathrm{M}}: M \rightarrow \mathbb{C}^{4}$ which is a dual helicity object according to some authors (see e.g., [21]) does satisfy 
the Dirac equation. However this statement is not correct. However an operator (quantum)Majorana field $\boldsymbol{\psi}_{\mathrm{M}}$ can satisfy Dirac equation if it is not a dual helicity object ( see Section 5.3). Also, even at a "classical level" a Majorana spinor field satisfies Dirac equation if for any $x \in M$ their components take values in a Grassmann algebra. Also, differently from the case of elko spinor fields some authors claim that Majorana fields are not dual helicities objects [1, a statement that is correct only for Majorana quantum fields as constructed in Section 5.3. For a Majorana field (even at "classical level") whose components take values in a Grassmann algebra the statement is not correct.

Finally, since according to our findings the elko spinor fields as well as the fields $\mathcal{K}$ and $\mathcal{M}$ are of mass dimension $3 / 2$ we show in Section 6 how to calculate the correct propagators for $\mathcal{K}$ and $\mathcal{M}$. We also show that the causal propagator for the covariant $\boldsymbol{\lambda}$ and $\boldsymbol{\rho}$ fields is simply the standard Feynman propagator of Dirac theory.

In presenting the above results we use the representation of spinor fields in the Clifford bundle formalism $(\mathrm{CBF})$ 23, 26, 31. This is briefly recalled in section 2 where a useful translation for the standard matrix formalism 1 to the $\mathrm{CBF}$ is given. The CBF makes all calculations easy and transparent and in particular permits to infer [27] in a while that elko spinor fields are class 5 spinor fields in Lounesto classification [20, 27.

\section{Description of Spinor Fields in the Clifford Bundle}

Let $\left(M \simeq \mathbb{R}^{4}, \eta, D, \tau_{\eta}\right)$ be the Minkowski spacetime structure where $\eta \in \sec T_{0}^{2} M$ is Minkowski metric and $D$ is the Levi-Civita connection of $\eta$. Also, $\tau_{\eta} \in$ $\sec \bigwedge^{4} T^{*} M$ defines an orientation. We denote by $\eta \in \sec T_{2}^{0} M$ the metric of the cotangent bundle. It is defined as follows. Let $\left\langle x^{\mu}\right\rangle$ be coordinates for $M$ in the Einstein-Lorentz-Poincaré gauge. Let $\left\langle\boldsymbol{e}_{\mu}=\partial / \partial x^{\mu}\right\rangle$ a basis for $T M$ and $\left\langle\gamma^{\mu}=d x^{\mu}\right\rangle$ the corresponding dual basis for $T^{*} M$, i.e., $\gamma^{\mu}\left(\boldsymbol{e}_{\alpha}\right)=\delta_{\alpha}^{\mu}$. Then, if $\eta=\eta_{\mu \nu} \gamma^{\mu} \otimes \gamma^{\nu}$ then $\eta=\eta^{\mu \nu} \boldsymbol{e}_{\mu} \otimes \boldsymbol{e}_{\nu}$, where the matrix with entries $\eta_{\mu \nu}$ and the one with entries $\eta^{\mu \nu}$ are the equal to the diagonal matrix $\operatorname{diag}(1,-1,-1,-1)$. If $a, b \in \sec \bigwedge^{1} T^{*} M$ we write $a \cdot b=\eta(a, b)$. We also denote by $\left\langle\gamma_{\mu}\right\rangle$ the reciprocal basis of $\left\langle\gamma^{\mu}=d x^{\mu}\right\rangle$, which satisfies $\gamma^{\mu} \cdot \gamma_{\nu}=\delta_{\nu}^{\mu}$.

We denote the Clifford bundle of differential form: 2 by $\mathcal{C} \ell(M, \eta)$ and use notations and conventions in what follows as in 31] and recall the fundamental

\footnotetext{
${ }^{1}$ If more details are need the reader may find the necessary help in [31].

${ }^{2}$ We recall that $\mathcal{C} \ell\left(T_{x}^{*} M, \eta\right) \simeq \mathbb{R}_{1,3}$ the so-called spacetime algebra. Also the even subalgebra of $\mathbb{R}_{1,3}$ denoted $\mathbb{R}_{1,3}^{0}$ is isomorphic to te Pauli algebra $\mathbb{R}_{3,0}$, i.e., $\mathbb{R}_{1,3}^{0} \simeq \mathbb{R}_{3,0}$. The even subalgebra of the Pauli algebra $\mathbb{R}_{3,0}^{0}:=\mathbb{R}_{3,0}^{00}$ is the quaternion algebra $\mathbb{R}_{0,2}$, i.e., $\mathbb{R}_{0,2} \simeq \mathbb{R}_{3,0}^{0}$. Moreover we have the identifications: $\operatorname{Spin}_{1,3}^{0} \simeq \operatorname{Sl}(2, \mathbb{C}), \operatorname{Spin}_{3,0} \simeq S U(2)$. For the Lie algebras of these groups we have $\operatorname{spin}_{1,3}^{0} \simeq \operatorname{sl}(2, \mathbb{C}), s u(2) \simeq \operatorname{spin}_{3,0}$. The important fact to keep in mind for the understanding of some of the identificastions we done below is that $\operatorname{Spin}_{1,3}^{0}, \operatorname{spin}_{1,3}^{0} \subset \mathbb{R}_{3,0} \subset \mathbb{R}_{1,3}$ and $\operatorname{Spin}_{3,0}, \operatorname{spin}_{3,0} \subset \mathbb{R}_{0,2} \subset \mathbb{R}_{1,3}^{0} \subset \mathbb{R}_{1,3}$. If more details are need the read should consult, e.g., 31.
} 
relation

$$
\gamma^{\mu} \gamma^{\nu}+\gamma^{\nu} \gamma^{\mu}=2 \eta^{\mu \nu}
$$

As well known all (covariant) spinor fields carrying a $(1 / 2,0) \oplus(0,1 / 2)$ representation of $\operatorname{Spin}_{1,3}^{0} \simeq \operatorname{Sl}(2, \mathbb{C})$ belongs to one of the six Lounesto classes [20]. As well known a $(1 / 2,0) \oplus(0,1 / 2)$ spinor field in Minkowski spacetime is an equivalence class of triplets $(\boldsymbol{\psi}, \Sigma, \Xi)$ where for each $x \in M, \boldsymbol{\psi}(x) \in \mathbb{C}^{4}, \Sigma$ is an orthonormal coframe and $\Xi=u \in \operatorname{Spin}_{1,3}^{0}(M, \eta) \subset \mathcal{C} \ell(M, \eta)$ is a spinorial frame. If we fix a fiducial global coframe $\Sigma_{0}=\left\langle\dot{\gamma}^{\mu}\right\rangle$ and take, e.g., $\Xi_{0}=u_{0}=$ $1 \in \operatorname{Spin}_{1,3}^{0}(M, \eta) \subset \mathcal{C} \ell(M, \eta)$ the triplet $\left(\boldsymbol{\psi}_{0}, \Sigma_{0}, \Xi_{0}\right)$ is equivalent to $(\psi, \Sigma, \Xi)$ if $\gamma^{\mu}=\Lambda_{\nu}^{\mu} \dot{\gamma}^{\nu}=( \pm u) \gamma^{\mu}\left( \pm u^{-1}\right)$ and $\boldsymbol{\psi}(x)=S(u) \boldsymbol{\psi}_{0}(x)$ where $S(u)$ is the standard $(1 / 2,0) \oplus(0,1 / 2)$ matrix representation of $\mathrm{Sl}(2, \mathbb{C})$. Dirac gamma matrices in standard and Weyl representations will be denoted by $\gamma^{\mu}$ and $\gamma^{\mu^{\prime}}$ and are not to be confused with the $\gamma^{\mu} \in \sec \bigwedge^{1} T^{*} M \hookrightarrow \mathcal{C} \ell(M, \eta)$. As well known the gamma matrices satisfy $\gamma^{\mu} \gamma^{\nu}+\gamma^{\nu} \gamma^{\mu}=2 \eta^{\mu \nu}$ and $\gamma^{\mu^{\prime}} \gamma^{\prime \nu}+\gamma^{\prime \nu} \gamma^{\prime \mu}=2 \eta^{\mu \nu}$. The relation between the $\gamma^{\mu}$ and the $\gamma^{\prime \mu}$ is given by

$$
\gamma^{\prime \mu}=S \gamma^{\mu} S^{-1}
$$

where 3

$$
S=\frac{1}{\sqrt{2}}\left(\begin{array}{cc}
\mathbf{1} & \mathbf{1} \\
\mathbf{1} & -\mathbf{1}
\end{array}\right) .
$$

A representation of a $(1 / 2,0) \oplus(0,1 / 2)$ spinor field in the Clifford bundle is an equivalence class of triplets $(\psi, \Sigma, \Xi)$ where $\psi \in \sec \mathcal{C} \ell^{0}(M, \eta)$ (the even subbundle of $\sec \mathcal{C} \ell(M, \eta)), \Sigma$ is an orthonormal coframe and $\Xi_{u}=u \in \operatorname{Spin}_{1,3}^{0}(M, \eta) \subset$ $\mathcal{C} \ell(M, \eta)$ is a spinorial frame. If we fix a fiducial global coframe $\Sigma_{0}=\left\langle\Gamma^{\mu}\right\rangle$ and take $\Xi_{u_{0}}=u_{0}=1 \in \sec \operatorname{Spin}_{1,3}^{0}(M, \eta) \subset \sec \mathcal{C} \ell(M, \eta)$ the triplet $\left(\psi_{0}, \Sigma_{0}, \Xi_{0}\right)$ is equivalent td $\left(\psi, \Sigma, \Xi_{u}\right)$ if $\gamma^{\mu}=\Lambda_{\nu}^{\mu} \Gamma^{\nu}=(u) \gamma^{\mu}\left(u^{-1}\right)$ and $\psi=\psi_{0} u^{-1}$. Field $\psi$ is called an operator spinor field and the operator spinor fields belonging to Lounesto classes 1,2,3 are also known as Dirac-Hestenes spinor fields.

If $\gamma^{\mu}, \mu=0,1,2,3$ are the Dirac gamma matrices in the standard representation and $\left\langle\gamma_{\mu}\right\rangle$ are as introduced above, we define

$$
\begin{aligned}
\sigma_{k} & :=\gamma_{k} \gamma_{0} \in \sec \bigwedge^{2} T^{*} M \hookrightarrow \sec \mathcal{C} \ell^{0}(M, \eta), k=1,2,3, \\
\mathbf{i} & =\gamma_{5}:=\gamma_{0} \gamma_{1} \gamma_{2} \gamma_{3} \in \sec \bigwedge^{4} T^{*} M \hookrightarrow \sec \mathcal{C} \ell(M, \eta), \\
\gamma_{5} & :=\gamma_{0} \gamma_{1} \gamma_{2} \gamma_{3} \in \operatorname{Mat}(4, \mathbb{C}) .
\end{aligned}
$$

Then, to the covariant spinor $\psi: M \rightarrow \mathbb{C}^{4}$ (in standard representation of the gamma matrices) where $\left(i=\sqrt{-1}, \phi, \varsigma: M \rightarrow \mathbb{C}^{2}\right)$

$$
\boldsymbol{\psi}=\left(\begin{array}{c}
\boldsymbol{\phi} \\
\varsigma
\end{array}\right)=\left(\begin{array}{c}
\left(\begin{array}{c}
m^{0}+i m^{3} \\
-m^{2}+i m^{1}
\end{array}\right) \\
\left(\begin{array}{c}
n^{0}+i n^{3} \\
-n^{2}+i n^{1}
\end{array}\right)
\end{array}\right),
$$

\footnotetext{
${ }^{3}$ We will supress the writing of the $4 \times 4$ and the $2 \times 2$ unity matrices when no confusion arises.

${ }^{4}$ Take notice that $\left(\psi, \Sigma, \Xi_{u}\right)$ is not equivalent to $\left(\psi, \Sigma, \Xi_{-u}\right)$ even if $(u) \gamma^{\mu}\left(u^{-1}\right)=$ $(-u) \gamma^{\mu}\left(-u^{-1}\right)$.
} 
there corresponds the operator spinor field $\psi \in \sec \mathcal{C} \ell^{0}(M, \eta)$ given by

$$
\psi=\phi+\varsigma \sigma_{3}=\left(m^{0}+m^{k} \mathbf{i} \sigma_{k}\right)+\left(n^{0}+n^{k} \mathbf{i} \sigma_{k}\right) \sigma_{3} .
$$

We then have the useful formulas in Eq. (9) below that one can use to immediately translate results of the standard matrix formalism in the language of the Clifford bundle formalism and vice-versa 5

$$
\begin{aligned}
\gamma_{\mu} \boldsymbol{\psi} & \leftrightarrow \gamma_{\mu} \psi \gamma_{0}, \\
i \boldsymbol{\psi} & \leftrightarrow \psi \gamma_{21}=\psi \mathbf{i} \sigma_{3}, \\
i \gamma_{5} \boldsymbol{\psi} & \leftrightarrow \psi \sigma_{3}=\psi \gamma_{3} \gamma_{0}, \\
\overline{\boldsymbol{\psi}} & =\boldsymbol{\psi}^{\dagger} \boldsymbol{\gamma}^{0} \leftrightarrow \tilde{\psi}, \\
\boldsymbol{\psi}^{\dagger} & \leftrightarrow \gamma_{0} \tilde{\psi} \gamma_{0}, \\
\boldsymbol{\psi}^{*} & \leftrightarrow-\gamma_{2} \psi \gamma_{2} .
\end{aligned}
$$

Remark 1 Note that $\gamma_{\mu}, i \mathbf{1}_{4}$ and the operations and $\dagger$ are for each $x \in M$ mappings $\mathbb{C}^{4} \rightarrow \mathbb{C}^{4}$. Then they are represented in the Clifford bundles formalism by extensor fields 31 which maps $\mathcal{C} \ell^{0}(M, \eta) \rightarrow \mathcal{C} \ell^{0}(M, \eta)$. Thus, to the operator $\gamma_{\mu}$ there corresponds an extensor field, call it $\underline{\gamma}_{\mu}: \mathcal{C} \ell^{0}(M, \eta) \rightarrow \mathcal{C} \ell^{0}(M, \eta)$ such that $\underline{\gamma}_{\mu} \psi=\gamma_{\mu} \psi \gamma_{0}$.

Using the above dictionary the standard Dirac equation 6 for a Dirac spinor field $\psi: M \rightarrow \mathbb{C}^{4}$

$$
i \gamma^{\mu} \partial_{\mu} \boldsymbol{\psi}-m \boldsymbol{\psi}=0
$$

translates immediately in the so-called Dirac-Hestenes equation, i.e.,

$$
\boldsymbol{\partial} \psi \gamma_{21}-m \psi \gamma_{0}=0
$$

Remark 2 In Eq.(11) the operator $\boldsymbol{\partial}$ acts on $\mathcal{C} \in \sec \mathcal{C} \ell(M, \eta)$ (when using the basis introduced above) a $\rrbracket^{7}$

$$
\left.\partial \mathcal{C}:=\gamma^{\mu}\right\lrcorner\left(\partial_{\mu} \mathcal{C}\right)+\gamma^{\mu} \wedge\left(\partial_{\mu} \mathcal{C}\right)
$$

Remark 3 It is sometimes useful, in particular when studying solutions for the Dirac-Hestenes equation to consider the Clifford bundle of multivector fields $\mathcal{C} \ell(M, \boldsymbol{\eta})$. We will write $\check{\psi} \in \sec \mathcal{C} \ell(M, \boldsymbol{\eta})$ for the sections of the $\mathcal{C} \ell(M, \boldsymbol{\eta})$ bundle. The Dirac-Hestenes equation in $\mathcal{C} \ell(M, \boldsymbol{\eta})$ is.

$$
\check{\boldsymbol{\partial}} \check{\psi} \boldsymbol{e}_{21}-m \check{\psi} \boldsymbol{e}_{0}=0 .
$$

where $\boldsymbol{e}_{\mu} \boldsymbol{e}_{\nu}+\boldsymbol{e}_{\nu} \boldsymbol{e}_{\mu}=2 \eta_{\mu \nu}$ and $\check{\boldsymbol{\partial}}:=\boldsymbol{e}^{\mu} \partial_{\mu}$ with $\boldsymbol{e}^{\mu}:=\eta^{\mu \nu}$ and(when using the basis introduced above)

$$
\left.\check{\partial} \check{\mathcal{C}}:=e^{\mu}\right\lrcorner\left(\partial_{\mu} \check{\mathcal{C}}\right)+e^{\mu} \wedge\left(\partial_{\mu} \check{\mathcal{C}}\right)
$$

\footnotetext{
${ }^{5} \tilde{\psi}$ is the reverse of $\psi$. If $A_{r} \in \sec \bigwedge^{r} T^{*} M \hookrightarrow \sec \mathcal{C} \ell(M, \eta)$ then $\tilde{A}_{r}=(-1)^{\frac{r}{2}(r-1)} A_{r}$.

${ }^{6} \partial_{\mu}:=\frac{\partial}{\partial x^{\mu}}$.

${ }^{7}$ The symbols $\lrcorner$ and $\wedge$ denote respctivley the leftcontraction and the exterior products in $\mathcal{C} \ell(M, \eta)$.
} 
for $\check{\mathcal{C}} \in \sec \mathcal{C} \ell(M, \boldsymbol{\eta})$. Keep in mind that in definition of $\check{\partial}$ the $\boldsymbol{e}^{\mu}$ are not supposed to act as a derivatives operators, i.e., $\left.\boldsymbol{e}^{\mu}\right\lrcorner\left(\partial_{\mu} \check{\mathcal{C}}\right)\left(\right.$ respectively $\left.\boldsymbol{e}^{\mu} \wedge\left(\partial_{\mu} \check{\mathcal{C}}\right)\right)$ is the left contraction of $\boldsymbol{e}^{\mu}$ with $\partial_{\mu} \check{\mathcal{C}}$ (respectively, the exterior product of $\boldsymbol{e}^{\mu}$ with $\left.\partial_{\mu} \mathcal{C}\right)$.

The basic positive and negative energy solutions of Eq.(10) which are eigenspinors of the helicity operator are 35]

$$
\mathbf{u}^{(1)}(\mathbf{p}) e^{-i p_{\mu} x^{\mu}}, \quad \mathbf{u}^{(2)}(\mathbf{p}) e^{-i p_{\mu} x^{\mu}}, \quad \mathbf{v}^{(1)}(\mathbf{p}) e^{i p_{\mu} x^{\mu}}, \quad \mathbf{v}^{(2)}(\mathbf{p}) e^{i p_{\mu} x^{\mu}} .
$$

The $\mathbf{u}^{(\alpha)}(\mathbf{p})$ and $\mathbf{v}^{(\alpha)}(\mathbf{p})(\alpha=1,2)$ are eigenspinors of the parity operatol $8 \mathbf{P}$, i.e.,

$$
\mathbf{P} \mathbf{u}^{(\alpha)}(\mathbf{p})=\mathbf{u}^{(\alpha)}(\mathbf{p}), \quad \mathbf{P} \mathbf{v}^{(\alpha)}(\mathbf{p})=\mathbf{v}^{(\alpha)}(\mathbf{p}),
$$

which makes Dirac equation invariant under a parity transformation?. These fields are represented in the Clifford bundle formalism by the following operator spinor fields,

$$
u^{(r)}(\mathbf{p})=L(\mathbf{p}) \varkappa^{(r)}, \quad v^{(r)}(\mathbf{p})=L(\mathbf{p}) \varkappa^{(r)} \sigma_{3},
$$

where $\varkappa^{(r)}=\left\{1,-\mathbf{i} \sigma_{2}\right\}$ and $L(\mathbf{p})$ is the following boost operator $(L(\mathbf{p}) \tilde{L}(\mathbf{p})=\mathbf{1})$

$$
L(\mathbf{p})=\frac{p \gamma^{0}+m}{\sqrt{2 m(E+m)}} .
$$

Remark 4 Recall that Dirac-Hestenes spinor fields couple to the electromagnetic potential $A \in \sec \bigwedge^{1} T^{*} M \hookrightarrow \sec \mathcal{C} \ell(M, \eta)$ as

$$
\boldsymbol{\partial} \psi \gamma_{21}-m \psi \gamma_{0}+e A \psi=0 .
$$

As it is well known this equation is invariant under a parity transformation of the fields $A$ and $\psi$.

In [1] the following (covariant) self and anti-self dual elko spinor fields $\boldsymbol{\lambda}_{\{+-\}}^{\prime s, a}$, $\boldsymbol{\lambda}_{\{-+\}}^{\prime s, a} \boldsymbol{\rho}_{\{+-\}}^{\prime s, a}, \boldsymbol{\rho}_{\{-+\}}^{\prime s, a}: M \rightarrow \mathbb{C}^{4}$ which are eigenspinors of the charge conjugation operator $(\mathbf{C})_{11}^{11}$ are defined using the Weyl,(chiral) representation of the gamma matrices by

$$
\begin{aligned}
& \boldsymbol{\lambda}_{\{\mp \pm\}}^{\prime s}(\mathbf{p})=\left(\begin{array}{c}
\sigma_{2}\left[\phi_{L}^{ \pm}(\mathbf{p})\right]^{*} \\
\phi_{L} \pm(\mathbf{p})
\end{array}\right), \quad \boldsymbol{\lambda}_{\{\mp \pm\}}^{\prime a}(\mathbf{p})=\left(\begin{array}{c}
-\sigma_{2}\left[\phi_{L}^{ \pm}(\mathbf{p})\right]^{*} \\
\phi_{L} \pm(\mathbf{p})
\end{array}\right), \\
& \boldsymbol{\rho}_{\{ \pm \mp\}}^{\prime s}(\mathbf{p})=\left(\begin{array}{c}
\phi_{R} \pm(\mathbf{p}) \\
-\sigma_{2}\left[\phi_{R}^{ \pm}(\mathbf{p})\right]^{*}
\end{array}\right), \quad \boldsymbol{\rho}_{\{ \pm \mp\}}^{a}(\mathbf{p})=\left(\begin{array}{c}
\phi_{R} \pm(\mathbf{p}) \\
\sigma_{2}\left[\phi_{R}^{ \pm}(\mathbf{p})\right]^{*}
\end{array}\right),
\end{aligned}
$$

\footnotetext{
${ }^{8}$ The parity operator acting on covariant spinor fields is defined as in 1 , i.e., $\mathbf{P}=i \gamma^{0} \mathcal{R}$, where $\mathcal{R}$ changes $\mathbf{p} \mapsto-\mathbf{p}$ and changes the eingenvalues of the helicity operator. For other possibilities for the parity operator, see e.g., page 50 of [7].

${ }^{9}$ For an easy and transparent way to see this rresult see Appendix .

${ }^{10}$ Recall that $p \gamma^{0}=p_{\mu} \gamma^{\mu} \gamma^{0}=E+\mathbf{p}$.

${ }^{11}$ The conjugation operator used in [1] is $\mathbf{C} \boldsymbol{\psi}=-\gamma^{2} \boldsymbol{\psi}^{*}$. Using the dictionary given by Eq. (9) we find that in the Clifford bundle formalism we have $\mathbf{C} \psi=-\psi \gamma_{20}$.
} 
where the $\mathbf{C} \boldsymbol{\lambda}^{\prime s}=+\boldsymbol{\lambda}^{\prime s}, \mathbf{C} \boldsymbol{\lambda}^{\prime a}=-\boldsymbol{\lambda}^{\prime a}$ and the indices $\{+-\},\{-+\}$ refers to the helicities of the upper and down components of the elko spinor fields, and where as in [1] we introduce the following helicity eigenstates 12 , $\phi_{L}^{+}(\mathbf{0})$ and $\phi_{L}^{-}(\mathbf{0})$ and $\phi_{R}^{+}(\mathbf{0})$ and $\phi_{R}^{-}(\mathbf{0})$ such that with $\hat{\mathbf{p}} \frac{\mathbf{p}}{|\mathbf{p}|}$ we have

$$
\begin{array}{ll}
\boldsymbol{\sigma} \cdot \frac{\mathbf{p}}{|\mathbf{p}|} \phi_{L}^{ \pm}(\mathbf{0}):= \pm \phi_{L}^{ \pm}(\mathbf{0}), & \boldsymbol{\sigma} \cdot \frac{\mathbf{p}}{|\mathbf{p}|}\left[\boldsymbol{\sigma}_{2}\left(\phi_{L}^{ \pm}(\mathbf{0})\right)^{*}\right]=\mp\left[\boldsymbol{\sigma}_{2}\left(\phi_{L}^{ \pm}(\mathbf{0})\right)^{*}\right], \\
\boldsymbol{\sigma} \cdot \frac{\mathbf{p}}{|\mathbf{p}|} \phi_{R}^{ \pm}(\mathbf{0}):= \pm \phi_{R}^{ \pm}(\mathbf{0}), & \boldsymbol{\sigma} \cdot \frac{\mathbf{p}}{|\mathbf{p}|}\left[-\boldsymbol{\sigma}_{2}\left(\phi_{R}^{ \pm}(\mathbf{0})\right)^{*}\right]=\mp\left[-\boldsymbol{\sigma}_{2}\left(\phi_{R}^{ \pm}(\mathbf{0})\right)^{*}\right]
\end{array}
$$

Also recall that being a general boost operator in the $D^{1 / 2,0} \oplus D^{0,1 / 2}$ representation of $S l(2, \mathbb{C})$

$$
\boldsymbol{K}=\boldsymbol{K}^{1 / 2,0} \oplus \boldsymbol{K}^{0,1 / 2}=e^{\frac{\sigma}{2} \cdot \alpha} \oplus e^{-\frac{\sigma}{2} \cdot \alpha}
$$

we have, e.g., taking $\alpha=\mathbf{p}$

$$
\boldsymbol{\lambda}_{\{-+\}}^{\prime s}(\mathbf{p})=\sqrt{\frac{E+m}{m}}\left(1-\frac{|\mathbf{p}|}{E+m}\right) \boldsymbol{\lambda}_{\{-+\}}^{\prime s}(\mathbf{0}),
$$

More details, if necessary, may be found in [1].

Remark 5 By dual helicity field we simply mean here that the formulas in Eq.(22) are satisfied. Note that the helicity operator (in both Weyl and standard representation of the gamma matrices) is

$$
\Sigma \cdot \frac{\mathbf{p}}{|\mathbf{p}|}=\left(\begin{array}{cc}
\sigma \cdot \frac{\mathbf{p}}{|\mathbf{p}|} & \mathbf{0} \\
\mathbf{0} & \sigma \cdot \frac{\mathbf{p}}{|\mathbf{p}|}
\end{array}\right) .
$$

$\mathbb{C}^{4}$-valued spinor fields depends for its definition of a choice of an inertial frame where the momentum of the particle is $\left(p_{0}, \mathbf{p}\right)$. The operator $\left(\boldsymbol{K}^{1 / 2,0} \oplus \boldsymbol{K}^{0,1 / 2}\right)$ commutes with $\boldsymbol{\sigma} \cdot \frac{\mathbf{p}}{|\mathbf{p}|}$ only if $\boldsymbol{\sigma} \cdot \boldsymbol{\alpha}$ is proportional to $\boldsymbol{\sigma} \cdot \frac{\mathbf{p}}{|\mathbf{p}|}$. So, the statement in [1] that the helicity operator commutes with the boost operator must be qualified. However, it remains true that $\sigma_{2}\left[\phi_{l}^{+}(\mathbf{p})\right]^{*}$ and $\phi_{l}^{+}(\mathbf{p})$ have opposite helicities for any $\mathbf{p}$.

Remark 6 Recall that, e.g., the $\mathbb{C}^{4}$-valued spinor field $\boldsymbol{\lambda}_{\{-+\}}^{\prime s}(\mathbf{p})$ given in the Weyl representation of the gamma matrices is represented by $\boldsymbol{\lambda}_{\{-+\}}^{s}(\mathbf{p})$ in the standard representation of the gamma matrices. We have

$$
\begin{aligned}
\boldsymbol{\lambda}_{\{-+\}}^{s}(\mathbf{p}) & =S \boldsymbol{\lambda}_{\{-+\}}^{\prime s}(\mathbf{p})=\frac{1}{\sqrt{2}}\left(\begin{array}{cc}
\mathbf{1} & \mathbf{1} \\
\mathbf{1} & -\mathbf{1}
\end{array}\right)\left(\begin{array}{c}
\sigma_{2}\left[\phi_{L}^{+}(\mathbf{p})\right]^{*} \\
\phi_{L}^{+}(\mathbf{p})
\end{array}\right) \\
& =\frac{1}{\sqrt{2}}\left(\begin{array}{c}
\sigma_{2}\left[\phi_{L}^{+}(\mathbf{p})\right]^{*}+\phi_{L}^{+}(\mathbf{p}) \\
\sigma_{2}\left[\phi_{L}^{+}(\mathbf{p})\right]^{*}-\phi_{L}^{+}(\mathbf{p})
\end{array}\right)
\end{aligned}
$$

\footnotetext{
${ }^{12}$ The indices $L$ and $R$ in $\phi_{L}^{ \pm}(\mathbf{p})$ and $\phi_{L}^{ \pm}(\mathbf{p})$ refer to the fact that these spinors fields transforms according to the basic non equivalent two dimensional representation of $\operatorname{Sl}(2, \mathbb{C})$.
} 
and then

$$
\boldsymbol{\Sigma} \cdot \frac{\mathbf{p}}{|\mathbf{p}|} \frac{1}{\sqrt{2}}\left(\begin{array}{c}
\sigma_{2}\left[\phi_{L}^{+}(\mathbf{p})\right]^{*}+\phi_{L}^{+}(\mathbf{p}) \\
\sigma_{2}\left[\phi_{L}^{+}(\mathbf{p})\right]^{*}-\phi_{L}^{+}(\mathbf{p})
\end{array}\right)=\frac{1}{\sqrt{2}}\left(\begin{array}{c}
-\sigma_{2}\left[\phi_{L}^{+}(\mathbf{p})\right]^{*}+\phi_{L}^{+}(\mathbf{p}) \\
-\sigma_{2}\left[\phi_{L}^{+}(\mathbf{p})\right]^{*}-\phi_{L}^{+}(\mathbf{p})
\end{array}\right)
$$

Remark 7 Recall that, e.g., the $\mathbb{C}^{4}$-valued spinor field $\boldsymbol{\lambda}_{\{-+\}}^{\prime s}(\mathbf{p})$ given in the Weyl representation of the gamma matrices is represented by $\boldsymbol{\lambda}_{\{-+\}}^{s}(\mathbf{p})$ in the standard representation of the gamma matrices. We have

$$
\begin{aligned}
\boldsymbol{\lambda}_{\{-+\}}^{s}(\mathbf{p}) & =S \boldsymbol{\lambda}_{\{-+\}}^{\prime s}(\mathbf{p})=\frac{1}{\sqrt{2}}\left(\begin{array}{cc}
\mathbf{1} & \mathbf{1} \\
\mathbf{1} & \mathbf{- 1}
\end{array}\right)\left(\begin{array}{c}
\sigma_{2}\left[\phi_{L}^{+}(\mathbf{p})\right]^{*} \\
\phi_{L}^{+}(\mathbf{p})
\end{array}\right) \\
& =\frac{1}{\sqrt{2}}\left(\begin{array}{l}
\sigma_{2}\left[\phi_{L}^{+}(\mathbf{p})\right]^{*}+\phi_{L}^{+}(\mathbf{p}) \\
\sigma_{2}\left[\phi_{L}^{+}(\mathbf{p})\right]^{*}-\phi_{L}^{+}(\mathbf{p})
\end{array}\right)
\end{aligned}
$$

and then

$$
\boldsymbol{\Sigma}^{\prime} \cdot \frac{\mathbf{p}}{|\mathbf{p}|} \frac{1}{\sqrt{2}}\left(\begin{array}{c}
\sigma_{2}\left[\phi_{L}^{+}(\mathbf{p})\right]^{*}+\phi_{L}^{+}(\mathbf{p}) \\
\sigma_{2}\left[\phi_{L}^{+}(\mathbf{p})\right]^{*}-\phi_{L}^{+}(\mathbf{p})
\end{array}\right)=\frac{1}{\sqrt{2}}\left(\begin{array}{c}
-\sigma_{2}\left[\phi_{L}^{+}(\mathbf{p})\right]^{*}+\phi_{L}^{+}(\mathbf{p}) \\
-\sigma_{2}\left[\phi_{L}^{+}(\mathbf{p})\right]^{*}-\phi_{L}^{+}(\mathbf{p})
\end{array}\right) .
$$

Eq.(28) and Eq.(29) show that the labels $\{-+\}$ (and also $\{+-\}$ ) as defining the helicities of the upper and down $\mathbb{C}^{2}$-valued components of a $\boldsymbol{\lambda}$ type spinor field in the standard representation of the gamma matrices have no meaning at all.

Also, one can make the identifications 13

$$
\begin{array}{lll}
\boldsymbol{\rho}_{\{+-\}}^{s}(\mathbf{p})=+i \boldsymbol{\lambda}_{\{+-\}}^{a}(\mathbf{p}), & \boldsymbol{\rho}_{\{-+\}}^{s}(\mathbf{p})=-i \boldsymbol{\lambda}_{\{-+\}}^{a}(\mathbf{p}), \\
\boldsymbol{\rho}_{\{+-\}}^{a}(\mathbf{p})=-i \boldsymbol{\lambda}_{\{+-\}}^{s}(\mathbf{p}), & \boldsymbol{\rho}_{\{-+\}}^{a}(\mathbf{p})=+i \boldsymbol{\lambda}_{\{-+\}}^{s}(\mathbf{p}) .
\end{array}
$$

Moreover, we recall that the elko spinor fields are not eigenspinors of the parity operator and indeed (see Eq.(4.14) and Eq.(4.15) in [1]),

$$
\begin{array}{ll}
\mathbf{P} \boldsymbol{\lambda}_{\{-+\}}^{s}(\mathbf{p})=+i \boldsymbol{\lambda}_{\{+-\}}^{a}(\mathbf{p})=\boldsymbol{\rho}_{\{+-\}}^{s}(\mathbf{p}), & \mathbf{P} \boldsymbol{\lambda}_{\{+-\}}^{s}(\mathbf{p})=-i \boldsymbol{\lambda}_{\{-+\}}^{a}(\mathbf{p})=\boldsymbol{\rho}_{\{-+\}}^{s}(\mathbf{p}), \\
\mathbf{P} \boldsymbol{\lambda}_{\{-+\}}^{a}(\mathbf{p})=-i \boldsymbol{\lambda}_{\{+-\}}^{s}(\mathbf{p})=\boldsymbol{\rho}_{\{+-\}}^{a}(\mathbf{p}), & \mathbf{P} \boldsymbol{\lambda}_{\{+-\}}^{a}(\mathbf{p})=+i \boldsymbol{\lambda}_{\{-+\}}^{s}(\mathbf{p})=\boldsymbol{\rho}_{\{-+\}}^{a}(\mathbf{p}) .
\end{array}
$$

Then if $\boldsymbol{\lambda}^{s, a}(x):=\boldsymbol{\lambda}^{s, a}(\mathbf{p}) \exp \left(\epsilon^{s, a} i p_{\mu} x^{\mu}\right)$, with $\epsilon^{s}=-1$ and $\epsilon^{a}=+1$ we have due to their construction that the elko spinor fields must satisfy the following csfopde:

$$
\begin{aligned}
& i \boldsymbol{\gamma}^{\mu} \partial_{\mu} \boldsymbol{\lambda}_{\{-+\}}^{s}+m \boldsymbol{\rho}_{\{+-\}}^{a}=0, \quad i \boldsymbol{\gamma}^{\mu} \partial_{\mu} \boldsymbol{\rho}_{\{-+\}}^{a}+m \boldsymbol{\lambda}_{\{+-\}}^{s}=0, \\
& i \gamma^{\mu} \partial_{\mu} \boldsymbol{\lambda}_{\{-+\}}^{a}-m \boldsymbol{\rho}_{\{+-\}}^{s}=0, \quad i \boldsymbol{\gamma}^{\mu} \partial_{\mu} \boldsymbol{\rho}_{\{-+\}}^{s}-m \boldsymbol{\lambda}_{\{+-\}}^{a}=0, \\
& i \gamma^{\mu} \partial_{\mu} \boldsymbol{\lambda}_{\{+-\}}^{s}-m \rho_{\{-+\}}^{a}=0, \quad i \gamma^{\mu} \partial_{\mu} \rho_{\{+-\}}^{a}-m \boldsymbol{\lambda}_{\{-+\}}^{s}=0, \\
& i \boldsymbol{\gamma}^{\mu} \partial_{\mu} \boldsymbol{\lambda}_{\{+-\}}^{a}+m \boldsymbol{\rho}_{\{-+\}}^{s}=0, \quad i \gamma^{\mu} \partial_{\mu} \boldsymbol{\rho}_{\{+-\}}^{s}+m \boldsymbol{\lambda}_{\{-+\}}^{a}=0 .
\end{aligned}
$$

${ }^{13}$ See Eq.(B.6) and Eq.(B.7) in [1]. 
If $\lambda_{\{+-\}}^{s, a}, \lambda_{\{-+\}}^{s, a}, \rho_{\{+-\}}^{s, a}, \rho_{\{-+\}}^{s, a} \in \sec \mathcal{C} \ell^{0}(M, \eta)$ are the representatives of the covariant spinors $\boldsymbol{\lambda}_{\{+-\}}^{s, a}, \boldsymbol{\lambda}_{\{-+\}}^{s, a}, \boldsymbol{\rho}_{\{+-\}}^{s, a}, \boldsymbol{\rho}_{\{-+\}}^{s, a}: M \rightarrow \mathbb{C}^{4}$ then they satisfy the csfopde:

$$
\begin{array}{ll}
\boldsymbol{\partial} \lambda_{\{-+\}}^{s} \gamma_{21}+m \rho_{\{+-\}}^{a} \gamma_{0}=0, & \boldsymbol{\partial} \rho_{\{-+\}}^{a} \gamma_{21}+m \lambda_{\{+-\}}^{s} \gamma_{0}=0, \\
\boldsymbol{\partial} \lambda_{\{-+\}}^{a} \gamma_{21}-m \rho_{\{+-\}}^{s} \gamma_{0}=0, & \boldsymbol{\partial} \rho_{\{-+\}}^{s} \gamma_{21}-m \lambda_{\{+-\}}^{a} \gamma_{0}=0, \\
\boldsymbol{\partial} \lambda_{\{+-\}}^{s} \gamma_{21}-m \rho_{\{-+\}}^{a} \gamma_{0}=0, & \boldsymbol{\partial} \rho_{\{+-\}}^{a} \gamma_{21}-m \lambda_{\{-+\}}^{s} \gamma_{0}=0, \\
\boldsymbol{\partial} \lambda_{\{+-\}}^{a} \gamma_{21}+m \rho_{\{-+\}}^{s} \gamma_{0}=0, & \boldsymbol{\partial} \rho_{\{+-\}}^{s} \gamma_{21}+m \lambda_{\{-+\}}^{a} \gamma_{0}=0 .
\end{array}
$$

Remark 8 From Eq.(33) it follows trivially that the operator spinor fields $\lambda_{\{+-\}}^{s, a}$,

$\lambda_{\{-+\}}^{s, a} \rho_{\{+-\}}^{s, a}, \rho_{\{-+\}}^{s, a} \in \sec \mathcal{C} \ell^{0}(M, \eta)$ satisfy Klein-Gordon equations. However, e.g., the Klein-Gordon equations

$$
\square \lambda_{\{-+\}}^{s}+m^{2} \lambda_{\{-+\}}^{s}=0, \quad \square \rho_{\{+-\}}^{a}+m^{2} \rho_{\{+-\}}^{a}=0,
$$

possess (as it is trivial to verify) solutions that are not solutions of the csfopde satisfied $\lambda_{\{-+\}}^{s}$ and $\rho_{\{+-\}}^{a}$. An immediate consequence of this observation is that attribution of mass dimension 1 to elko spinor fields seems equivocated. elko spinor fields as Dirac spinor fields have mass dimension 3/2, and the equation of motion for the elkos can be obtained from a Lagrangian (where the mass dimension of the fields are obvious) as we recall next.

\section{Lagrangian for the csfopde for the elko Spinor Fields}

A (multiform) Lagrangian that gives the Eqs. (33) for the operator elko spinor fields $\lambda_{\{-+\}}^{s}, \lambda_{\{-+\}}^{a}, \rho_{\{+-\}}^{a}, \rho_{\{-+\}}^{s} \in \sec \mathcal{C} \ell^{0}(M, \eta)$ having mass dimension $3 / 2$ is:

$\mathcal{L}=\frac{1}{2}\left\{\begin{array}{c}\left(\boldsymbol{\partial} \lambda_{\{+-\}}^{s} \mathbf{i} \gamma_{3}\right) \cdot \lambda_{\{+-\}}^{s}+\left(\boldsymbol{\partial} \lambda_{\{-+\}}^{a} \mathbf{i} \gamma_{3}\right) \cdot \lambda_{\{-+\}}^{a}+\left(\boldsymbol{\partial} \rho_{\{+-\}}^{a} \mathbf{i} \gamma_{3}\right) \cdot \rho_{\{+-\}}^{a} \\ +\left(\boldsymbol{\partial} \rho_{\{-+\}}^{s} \gamma \mathbf{i} \gamma_{3}\right) \cdot \rho_{\{-+\}}^{s}-2 m \lambda_{\{+-\}}^{s} \cdot \rho_{\{-+\}}^{a}+2 m \lambda_{\{-+\}}^{a} \cdot \rho_{\{+-\}}^{s}\end{array}\right\}$

The Euler-Lagrange equation obtained, e.g., from the variation of the field $\lambda_{\{+-\}}^{S}$ is: 14 :

$$
\partial_{\lambda_{\{+-\}}^{S}} \mathcal{L}-\boldsymbol{\partial}\left(\partial_{\partial \lambda_{\{+-\}}^{s}} \mathcal{L}\right)=0
$$

\footnotetext{
${ }^{14}$ See details and the definition of the multiform derivatives $\partial_{\lambda_{\{-+\}}^{s}}$ and $\partial_{\partial \lambda_{\{-+\}}^{s}}$ in Chapters 2 and 7 of 31.
} 
We have immediately $\sqrt{15}$

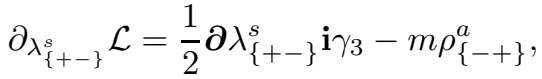

$$
\begin{aligned}
& \left.\partial_{\partial \lambda_{\{-+\}}^{s}} \mathcal{L}=-\frac{1}{2} \partial_{\partial \lambda_{\{+-\}}^{s}}\left(\partial \lambda_{\{-+\}}^{s}\right) \cdot \lambda_{\{+-\}}^{s} \mathbf{i} \gamma_{3}\right)=-\frac{1}{2} \lambda_{\{+-\}}^{s} \mathbf{i} \gamma_{3}, \\
& -\boldsymbol{\partial}\left(\partial_{\partial \lambda_{\{+-\}}^{s}} \mathcal{L}\right)=+\frac{1}{2} \partial \lambda_{\{+-\}}^{s} \mathbf{i} \gamma_{3} .
\end{aligned}
$$

Recalling that $\mathbf{i} \gamma_{3}=-\gamma_{0} \gamma_{1} \gamma_{2}$ the resulting Euler-Lagrange equation is

$$
\partial \lambda_{\{+-\}}^{s} \gamma_{21}-m \rho_{\{-+\}}^{a} \gamma_{0}=0 .
$$

Remark 9 With this result and the one in [10] we must say that the main claims concerning the attributes of elko spinor fields appearing in recent literature seems to us equivocated and the question arises: which kind of particles are described by these fields and to which gauge field do they couple? This question is answered in the next section.

\section{Coupling of the elko Spinor Fields a $s u(2) \simeq$ $\operatorname{spin}_{3,0}$ valued Potential $\mathcal{A}$}

We start by introducing Clifford valued differential multiforms fields, i.e., the objects

$$
\begin{aligned}
\mathcal{K} & =\lambda_{\{-+\}}^{s} \otimes 1-\rho_{\{+-\}}^{a} \otimes \mathfrak{i} \tau_{2} \in \sec \mathcal{C} \ell^{0}(M, \eta) \otimes \mathbb{R}_{1,3}^{0} \subset \sec \mathcal{C} \ell(M, \eta) \otimes \mathbb{R}_{1,3}^{0} \\
\mathcal{M} & =\lambda_{\{-+\}}^{s} \otimes 1-\rho_{\{+-\}}^{a} \otimes \mathfrak{i} \tau_{2} \in \sec \mathcal{C} \ell^{0 \subset}(M, \eta) \otimes \mathbb{R}_{1,3}^{0} \subset \sec \mathcal{C} \ell(M, \eta) \otimes \mathbb{R}_{1,3}^{0}
\end{aligned}
$$

where $\tau_{\mathbf{1}}, \tau_{\mathbf{2}}, \tau_{\mathbf{3}}$ are the generators of the Pauli algebra $\mathbb{R}_{3,0} \simeq \mathbb{R}_{1,3}^{0}$ and $\mathfrak{i}:=$ $\tau_{1} \tau_{\mathbf{2}} \tau_{\mathbf{3}}$. So, we have $\tau_{\mathbf{i}}:=\Gamma_{\mathbf{i}} \Gamma_{0}$ where the $\Gamma_{\mu}$ are the generators of $\mathbb{R}_{1,3}$, i.e., $\Gamma_{\mu} \Gamma_{\nu}+\Gamma_{\nu} \Gamma_{\mu}=2 \eta_{\mu \nu}$. Also, $i:=\tau_{1} \tau_{2} \tau_{3}=\Gamma_{0} \Gamma_{1} \Gamma_{2} \Gamma_{3}=: \Gamma_{5}$.

We define the reverse a general Clifford valued differential multiforms field

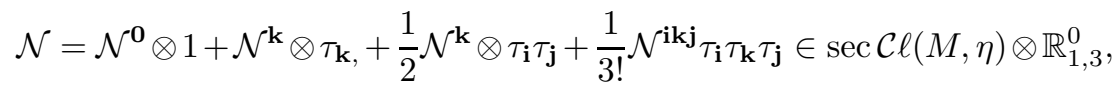

where $\mathcal{N}^{\mathbf{0}}, \mathcal{N}^{\mathbf{k}}, \mathcal{N}^{\mathbf{k}}, \mathcal{N}^{\mathbf{i k j}} \in \sec \mathcal{C} \ell(M, \eta)$ by

$$
\widetilde{\mathcal{N}}=\tilde{\mathcal{N}}^{\mathbf{0}} \otimes 1+\tilde{\mathcal{N}}^{\mathbf{k}} \otimes \tau_{\mathbf{k}},+\frac{1}{2} \widetilde{\mathcal{N}}^{\mathbf{i j}} \otimes \tau_{\mathbf{j}} \tau_{\mathbf{i}}+\frac{1}{3 !} \tilde{\mathcal{N}}^{\mathbf{i j k}} \tau_{\mathbf{k}} \tau_{\mathbf{j}} \tau_{\mathbf{i}}
$$

Since, as well known the $\tau_{\mathbf{1}}, \tau_{\mathbf{2}}, \tau_{\mathbf{3}}$ have a matrix representation in $\mathbb{C}(2)$, namely $\boldsymbol{\tau}_{\mathbf{1}}, \boldsymbol{\tau}_{\mathbf{2}}, \boldsymbol{\tau}_{\mathbf{3}}$, a set of Pauli matrices, we have the correspondences

$$
\mathcal{K} \leftrightarrow\left(\begin{array}{cc}
\lambda_{\{-+\}}^{s} & -\rho_{\{+-\}}^{a} \\
\rho_{\{+-\}}^{a} & \lambda_{\{-+\}}^{s}
\end{array}\right), \quad \mathcal{M} \leftrightarrow\left(\begin{array}{cc}
\lambda_{\{+-\}}^{s} & -\rho_{\{-+\}}^{a} \\
\rho_{\{-+\}}^{a} & \lambda_{\{+-\}}^{s}
\end{array}\right)
$$

\footnotetext{
${ }^{15}$ In the second line of Eq. (37) we used the identity $(K L) \cdot M=K \cdot(M \tilde{L})$ for all $K, L, M \in$ $\sec \mathcal{C} \ell(M, \eta)$.
} 
We observe moreover that

$$
\mathbf{K}=\mathcal{K} \frac{1}{2}\left(1+\tau_{\mathbf{3}}\right) \leftrightarrow\left(\begin{array}{cc}
\lambda_{\{-+\}}^{s} & 0 \\
\rho_{\{+-\}}^{a} & 0
\end{array}\right), \mathbf{M}=\frac{1}{2} \mathcal{K} \frac{1}{2}\left(1+\tau_{\mathbf{3}}\right) \leftrightarrow\left(\begin{array}{cc}
\lambda_{\{+-\}}^{s} & 0 \\
\rho_{\{-+\}}^{a} & 0
\end{array}\right)
$$

Then, from Eqs. (33) we can show that the $\mathcal{K}$ and $\mathcal{M}$ fields satisfy the following linear partial differential equations

$$
\begin{aligned}
\partial \mathcal{K} \gamma_{21}-m \mathcal{K} \mathfrak{i} \tau_{2} \gamma_{0} & =0 \\
\partial \mathcal{M} \gamma_{21}+m \mathcal{M i} \tau_{2} \gamma_{0} & =0
\end{aligned}
$$

Indeed, $\mathcal{K} \mathfrak{i} \tau_{2}=\mathfrak{i} \tau_{2} \mathcal{K}=\lambda_{\{-+\}}^{s} \otimes \mathfrak{i} \tau_{2}-\rho_{\{+-\}}^{a} \otimes 1, \mathcal{M i} \tau_{2}=\mathcal{M i} \tau_{2}=\lambda_{\{-+\}}^{s} \otimes$ $\mathfrak{i} \tau_{2}-\rho_{\{-+\}}^{a} \otimes 1$ and we have the correspondences:

$$
\begin{aligned}
\mathcal{K} \leftrightarrow\left(\begin{array}{cc}
\lambda_{\{-+\}}^{s} & -\rho_{\{+-\}}^{a} \\
\rho_{\{+-\}}^{a} & \lambda_{\{-+\}}^{s}
\end{array}\right), \quad \mathcal{M} \leftrightarrow\left(\begin{array}{cc}
\lambda_{\{+-\}}^{s} & -\rho_{\{-+\}}^{a} \\
\rho_{\{-+\}}^{a} & \lambda_{\{+-\}}^{s}
\end{array}\right), \\
\mathcal{K} \mathfrak{i} \tau_{\mathbf{2}}=\mathfrak{i} \tau_{\mathbf{2}} \mathcal{K} \leftrightarrow\left(\begin{array}{cc}
\rho_{\{+-\}}^{a} & \lambda_{\{-+\}}^{s} \\
-\lambda_{\{-+\}}^{s} & \rho_{\{+-\}}^{a}
\end{array}\right), \quad \mathcal{M i} \tau_{2}=\mathfrak{i} \tau_{2} \mathcal{M} \leftrightarrow\left(\begin{array}{cc}
\rho_{\{-+\}}^{a} & \lambda_{\{+-\}}^{s} \\
-\lambda_{\{+-\}}^{s} & \rho_{\{-+\}}^{a}
\end{array}\right)
\end{aligned}
$$

Then, from Eqs.(43) and (44) we see that $\mathbf{K}$ and $\mathbf{M}$ satisfy the following linear partial differential equations

$$
\begin{aligned}
\partial \mathbf{K} \gamma_{21}+\mathfrak{i} m \tau_{\mathbf{2}} \mathbf{K} \gamma_{0} & =0 \\
\partial \mathbf{M} \gamma_{21}-\mathfrak{i} m \tau_{\mathbf{2}} \mathbf{M} \gamma_{0} & =0
\end{aligned}
$$

which, on taking the corresponding matrix representation gives the coupled equations for the pairs $\left(\lambda_{\{-+\}}^{s}, \rho_{\{+-\}}^{a}\right)$ and $\left(\lambda_{\{+-\}}^{s}, \rho_{-+\}}^{a}\right)$ appearing in Eqs.(33).

Before proceeding we observe that the currents

$$
\begin{aligned}
\boldsymbol{J}_{\mathcal{K}} & =\mathcal{K} \tau_{\mathbf{1}} \gamma_{0} \widetilde{\mathcal{K}} \in \sec \bigwedge^{1} T^{*} M \otimes \operatorname{spin}_{3,0} \hookrightarrow \sec \mathcal{C} \ell(M, \eta) \otimes \mathbb{R}_{1,3}^{0}, \\
\boldsymbol{J}_{\mathcal{M}} & =\mathcal{M} \tau_{\mathbf{1}} \gamma_{0} \widetilde{\mathcal{M}} \in \sec \bigwedge^{1} T^{*} M \otimes \operatorname{spin}_{3,0} \hookrightarrow \sec \mathcal{C} \ell(M, \eta) \otimes \mathbb{R}_{1,3}^{0},
\end{aligned}
$$

are conserved, i.e.,

$$
\left.\boldsymbol{\partial}\lrcorner \boldsymbol{J}_{\mathcal{K}}=0, \quad \boldsymbol{\partial}\right\lrcorner \boldsymbol{J}_{\mathcal{M}}=0 .
$$

Indeed, let us show that $\boldsymbol{\partial}\lrcorner \boldsymbol{J}_{\mathcal{K}}=0$. We have

$$
\boldsymbol{\partial}\lrcorner \boldsymbol{J}_{\mathcal{K}}=\frac{1}{2}\left(\boldsymbol{\partial} \mathcal{K} \tau_{\mathbf{1}} \gamma_{0} \tilde{\mathcal{K}}+\mathcal{K} \tau_{\mathbf{1}} \gamma_{0} \tilde{\mathcal{K}} \overleftarrow{\boldsymbol{\partial}}\right)
$$

From Eq.(43) we have

$$
\partial \mathcal{K}=\mathfrak{i} m \mathcal{K}_{\tau_{2}} \gamma_{012}, \quad \widetilde{\mathcal{K}} \overleftarrow{\boldsymbol{\partial}}=\partial_{\mu} \widetilde{\mathcal{K}} \gamma^{\mu}=\mathfrak{i} m \gamma_{012} \tau_{2} \widetilde{\mathcal{K}}
$$


Then,

$$
\begin{aligned}
\boldsymbol{\partial}\lrcorner \boldsymbol{J}_{\mathcal{K}} & =\frac{1}{2}\left(\mathfrak{i} m \mathcal{K} \tau_{\mathbf{2}} \gamma_{012} \tau_{\mathbf{1}} \gamma_{0} \widetilde{\mathcal{K}}+\mathfrak{i} m \mathcal{K} \gamma_{12} \tau_{\mathbf{1}} \tau_{\mathbf{2}} \widetilde{\mathcal{K}}\right) \\
& =\frac{\mathfrak{i} m}{2}\left(\mathcal{K}\left(\tau_{\mathbf{2}} \tau_{\mathbf{1}}+\tau_{\mathbf{1}} \tau_{\mathbf{2}}\right) \gamma_{12} \widetilde{\mathcal{K}}=0 .\right.
\end{aligned}
$$

The fields $\mathcal{K}$ and $\mathcal{M}$ are electrically neutral, but they can couple with an $s u(2) \simeq \operatorname{spin}_{3,0} \subset \mathbb{R}_{3,0}$ valued potential

$$
\mathcal{A}=A^{\mathbf{i}} \otimes \tau_{\mathbf{i}} \in \sec \bigwedge^{1} T^{*} M \otimes \operatorname{spin}_{3,0} \hookrightarrow \sec \mathcal{C} \ell(M, \eta) \otimes \mathbb{R}_{1,3}
$$

Indeed, we have taking into account that $\mathfrak{i}=\Gamma_{\mathbf{5}}, \tau_{\mathbf{i}}=\Gamma_{\mathbf{i} 0}$ that the coupling is

$$
\begin{aligned}
\partial \mathcal{K} \gamma_{21}-m \mathcal{K}-{ }_{5}-{ }_{20} \gamma_{0}+q-{ }_{5} \mathcal{A K} & =0, \\
\partial \mathcal{M} \gamma_{21}+m \mathcal{M}-{ }_{5}-{ }_{20} \gamma_{0}+q-{ }_{5} \mathcal{A M} & =0 .
\end{aligned}
$$

Equations (54) and (55) are invariant under the following transformation of the fields and change of the basis of the $\operatorname{spin}_{3,0} \subset \mathbb{R}_{1,3}^{00}$ algebra:

$$
\begin{aligned}
\mathcal{K} \mapsto \mathcal{K}^{\prime}=e^{\Gamma_{5} q \theta^{\mathrm{i}} \Gamma_{\mathbf{i} 0}} \mathcal{K}, & \mathcal{M} \mapsto \mathcal{M}^{\prime}=e^{-{ }_{5} q \theta^{\mathrm{i}}-\mathrm{i} 0} \mathcal{M}, \\
\mathcal{A} \mapsto \mathcal{A}^{\prime}=e^{-{ }_{5} q \theta^{\mathrm{i}}-\mathrm{i} 0} \mathcal{A} e^{--{ }_{5} q \theta^{\mathrm{i}}-\mathrm{i} 0}, & \Gamma_{\mathbf{i}} \mapsto \Gamma_{\mathbf{i}}^{\prime}=e^{-5 q \theta^{\mathrm{i}}-\mathrm{i} 0} \Gamma_{\mathbf{i}} e^{-{ }_{5} q \theta^{\mathrm{i}}-\mathrm{i} 0}
\end{aligned}
$$

With the above result we propose that elko spinor fields of the $\lambda$ and $\rho$ types,are the crucial ingredients permitting the existence of the $\mathcal{K}$ and $\mathcal{M}$ fields which do not carry electric charges but possess magnetid 16 like charges that couple to an $\operatorname{spin}_{3,0} \subset \mathbb{R}_{1,3}^{00}$ valued potential $\mathcal{A}$.

\section{Difference Between Elko and Majorana Spinor Fields}

Here we recall that a Majorana field (also in class five in Lounesto classification 17 and supposedly describing a Majorana neutrino) differently from an elko spinor field is supposed in some textbooks to satisfy the Dirac equation (see, e.g., 21]), even if that equation cannot be derived from a Lagrangian (unless, as it is well known the components of Majorana fields for each $x \in M$ are Grassmann 'numbers'). The "proof" in 21 for the statement that a Majorana field $\boldsymbol{\psi}_{\mathbf{M}}^{\prime}$ : $M \rightarrow \mathbb{C}^{4}$ satisfies the Dirac equation is as follows. That author writes that

\footnotetext{
${ }^{16}$ The use of the term magnetic like charge here comes from the analogy to the possibel coupling of Weyl fields describing massless magnetic monoples with the electromagnetic potential $A \in \sec \wedge^{1} T^{*} M$. See 30 31.

${ }^{17}$ We mention Dirac spinor fields are the real type fermion fields and that Majorana and Elko spinor fields are the imaginary type fermion fields according to Yang and Tiomno [38] classification of spinor fields according to their transformation laws under parity .
} 
$\phi_{r}: M \rightarrow \mathbb{C}^{2}$ and $\phi_{l}: M \rightarrow \mathbb{C}^{2}$ belonging respectively to the carrier spaces of the representations $D^{0,1 / 2}$ and $D^{1 / 2,0}$ of $S l(2, \mathbb{C})$ satisfy

$$
\begin{aligned}
\boldsymbol{\sigma}^{\mu} i \partial_{\mu} \phi_{r} & =m \phi_{l}, \\
\breve{\boldsymbol{\sigma}}^{\mu} i \partial_{\mu} \phi_{l} & =m \phi_{r},
\end{aligned}
$$

with $\boldsymbol{\sigma}^{\mu}=\left(\mathbf{1}, \boldsymbol{\sigma}^{i}\right)$ and $\breve{\boldsymbol{\sigma}}^{\mu}=\left(\mathbf{1},-\boldsymbol{\sigma}^{i}\right)$ where $\boldsymbol{\sigma}^{i}\left(=\boldsymbol{\sigma}_{i}\right)$ are the Pauli matrices. From this we can see that we can write:

$$
i\left(\begin{array}{cc}
\mathbf{0} & \breve{\boldsymbol{\sigma}}^{\mu} \\
\boldsymbol{\sigma}^{\mu} & \mathbf{0}
\end{array}\right) \partial_{\mu}\left(\begin{array}{c}
\phi_{r} \\
\phi_{l}
\end{array}\right)=m\left(\begin{array}{c}
\phi_{r} \\
\phi_{l}
\end{array}\right) .
$$

The set of matrices $\gamma^{\prime \mu}:=\left(\begin{array}{cc}\mathbf{0} & \breve{\boldsymbol{\sigma}}^{\mu} \\ \boldsymbol{\sigma}^{\mu} & \mathbf{0}\end{array}\right)$ is ( as well known) a representation of Dirac matrices in Weyl representation,. It follows that $\boldsymbol{\psi}^{\prime}$ satisfy the Dirac equation, i.e.,

$$
i \gamma^{\prime \mu} \partial_{\mu} \psi^{\prime}-m \psi^{\prime}=0 .
$$

Have saying that, 21] defines a Majorana field (in Weyl representation) by

$$
\psi_{\mathbf{M}}^{\prime}=\left(\begin{array}{c}
\phi_{l} \\
\phi_{r}
\end{array}\right)=\left(\begin{array}{c}
\phi_{l} \\
i \sigma^{2} \phi_{l}^{*}
\end{array}\right),
$$

and write

$$
i \gamma^{\prime \mu} \partial_{\mu} \psi_{\mathbf{M}}^{\prime}-m \boldsymbol{\psi}_{\mathbf{M}}^{\prime}=0
$$

concluding his "proof".

Now, let us investigate more deeply that "proof". First recall that writing

$$
\phi_{r}(x)=\phi_{r}(\mathbf{p}) e^{\mp i p_{\mu} x^{\mu}}, \quad \phi_{l}(x)=\phi_{l}(\mathbf{p}) e^{\mp i p_{\mu} x^{\mu}},
$$

we have from Eq.(157) and Eq.(158) that

$$
\begin{aligned}
\left(p_{0}-\boldsymbol{\sigma} \cdot \mathbf{p}\right) \phi_{r}(\mathbf{p}) & = \pm m \phi_{l}(\mathbf{p}), \\
\left(p_{0}+\boldsymbol{\sigma} \cdot \mathbf{p}\right) \phi_{l}(\mathbf{p}) & = \pm m \phi_{r}(\mathbf{p}) .
\end{aligned}
$$

However, if $\phi_{l}(\mathbf{0})$ and $\phi_{r}(\mathbf{0})$ are the zero momentum fields we have (with $\varkappa$ being the boost parameter, i.e., $\sinh \varkappa / 2=\sqrt{(\gamma-1) / 2)}$ with $\gamma=1 / \sqrt{1-v^{2}}$ and $\mathbf{n}$ the direction of motion) by definition:

$$
\begin{aligned}
& \phi_{r}(\mathbf{p}):=e^{\frac{1}{2} \varkappa \cdot \boldsymbol{\sigma}} \phi_{r}(\mathbf{0})=(\cosh \varkappa / 2+\boldsymbol{\sigma} \cdot \mathbf{n} \sinh \varkappa / 2)=\frac{p_{0}+m+\boldsymbol{\sigma} \cdot \mathbf{p}}{\left[2 m\left(p_{0}+m\right)\right]^{1 / 2}} \phi_{r}(\mathbf{0}), \\
& \phi_{l}(\mathbf{p}):=e^{-\frac{1}{2} \varkappa \cdot \boldsymbol{\sigma}} \phi_{l}(\mathbf{0})=(\cosh \varkappa / 2-\boldsymbol{\sigma} \cdot \mathbf{n} \sinh \varkappa / 2) \phi_{l}(\mathbf{0})=\frac{p_{0}+m-\boldsymbol{\sigma} \cdot \mathbf{p}}{\left[2 m\left(p_{0}+m\right)\right]^{1 / 2}} \phi_{l}(\mathbf{0}) .
\end{aligned}
$$



i 18

We can now verify that Eq.(66) and Eq.(67) only imply Eq.(64) and Eq.(65)

$$
\phi_{l}(\mathbf{0})= \pm \phi_{r}(\mathbf{0})
$$

But this condition cannot be satisfied for a Majorana field $\psi_{\mathrm{M}}^{\prime}: M \rightarrow \mathbb{C}^{4}$ as defined by 21] where $\phi_{r}(\mathbf{0})=i \boldsymbol{\sigma}^{2} \phi_{l}^{*}(\mathbf{0})$. Indeed, writing $\phi_{l}^{t}(\mathbf{0})=(\nu, w)$ with $v, w \in \mathbb{C}$ we see that to have $\phi_{l}(\mathbf{0})= \pm \phi_{r}(\mathbf{0})$ we need $\nu=\omega^{*}$ and $\omega=-\nu^{*}$, i.e., $\nu=\omega=0$. We conclude that a Majorana field $\psi_{\mathbf{M}}^{\prime}: M \rightarrow \mathbb{C}^{4}$ cannot satisfy the Dirac equation.

\subsection{Some Majorana Fields are Dual Helicities Objects}

Before continuing we recall also that it is a well known fact (see, e.g., [16]) that the Dirac Hamiltonian commutes with the operator $\boldsymbol{\Sigma} \cdot \hat{\mathbf{p}}$ given by Eq.(25). Thus any $\Psi: M \rightarrow \mathbb{C}^{4}$ satisfying Dirac equation which is an eigenspinor of the Dirac Hamiltonian may be constructed such that $\phi_{l}$ and $\phi_{r}$ have the same helicity. Since a Majorana spinor field $\psi_{M}^{\prime}: M \rightarrow \mathbb{C}^{4}$ as defined by 21] does not satisfy Dirac equation we may suspect that it is not an eigenspinor of the of the operator $\boldsymbol{\Sigma} \cdot \hat{\mathbf{p}}$. And indeed this is the case, for we now show $\phi_{l}(\mathbf{0})$ and $\phi_{r}(\mathbf{0})$ in a Majorana field $\boldsymbol{\psi}_{\mathbf{M}}^{\prime}: M \rightarrow \mathbb{C}^{4}$ are not equal. Taking the momentum (without loss of generality) in the direction of the $z$-axis (of an inertial frame) and $\phi_{l}^{t}(\mathbf{0})=(1,0)$ we have

$$
\boldsymbol{\sigma} \cdot \hat{\mathbf{p}} \phi_{l}(\mathbf{0})=-\phi_{l}(\mathbf{0}), \quad \boldsymbol{\sigma} \cdot \hat{\mathbf{p}}\left(i \boldsymbol{\sigma}^{2} \phi_{l}(\mathbf{0})\right)=-i \boldsymbol{\sigma}^{2} \phi_{l}(\mathbf{0}),
$$

and as the elko spinor fields they are also dual helicities objects.

Remark 10 Keep also in mind that as well known even if a Majorana field is described by a field [22] $\varphi: M \rightarrow \mathbb{C}^{2}$ carrying the $D^{1 / 2,0}$ (or $D^{0,1 / 2}$ ) representation of $\operatorname{Sl}(2, \mathbb{C})$ the value of the helicity obviously depends on the inertial reference frame where the measurement is done [7, 24, because the helicity is invariant only under those Lorentz transformations which did not alter the direction of $\mathbf{p}$ along which the angular momentum component is taken.

\subsection{The Majorana Currents $J_{M}$ and $J_{M}^{5}$}

We observe moreover that if a Majorana field $\psi_{\mathrm{M}}^{\prime}: M \rightarrow \mathbb{C}^{4}$ should satisfy the Dirac equation then Eq.(62) should translate in the Clifford bundle formalism as

$$
\boldsymbol{\partial} \psi_{\mathbf{M}} \gamma_{21}-m \psi_{\mathbf{M}} \gamma_{0}=0
$$

where $\psi_{\mathbf{M}} \in \sec \mathcal{C} \ell^{0}(M, \eta)$. Then, current $\boldsymbol{J}_{\mathbf{M}}=\psi_{\mathbf{M}} \gamma_{0} \tilde{\psi}_{\mathbf{M}} \in \sec \bigwedge^{1} T^{*} M \hookrightarrow$ $\sec \mathcal{C} \ell(M, \eta)$ is conserved as it is trivial to verify. Moreover, it is lightlike (since for a class five spinor field $\tilde{\psi}_{\mathbf{M}} \psi_{\mathbf{M}}=0$ and thus $\boldsymbol{J}_{\mathbf{M}} \cdot \boldsymbol{J}_{\mathbf{M}}=\psi_{\mathbf{M}} \gamma_{0}\left(\tilde{\psi}_{\mathbf{M}} \psi_{\mathbf{M}}\right) \gamma_{0} \tilde{\psi}_{\mathbf{M}}=$

\footnotetext{
${ }^{18}$ That $\phi_{l}(\mathbf{0})= \pm \phi_{r}(\mathbf{0})$ is a necessary condition for a spinor field $\boldsymbol{\psi}: M \rightarrow \mathbb{C}^{4}$ to satisfy Dirac equation can bee seem, e.g., from Eq.(2.85) and Eq.(2.86) in Ryder's book [32]. However, Ryder misses the possible solution $\phi_{l}(\mathbf{0})=-\phi_{r}(\mathbf{0})$. This has been pointed by Ahluwalia 3 in his review of Ryder's book.
} 
0) but it is a non null covector field if the components of the spinor field $\boldsymbol{\psi}_{M}^{\prime}: M \rightarrow \mathbb{C}^{4}$ have values in $\mathbb{C}^{2}$. Indeed writing $\boldsymbol{J}_{\mathbf{M}}=\psi_{\mathbf{M}} \gamma_{0} \tilde{\psi}_{\mathbf{M}}=J_{\mathbf{M}}^{\mu} \gamma_{\mu}$ we see immediately that

$$
J_{M}^{0}=\bar{\psi}_{M}^{\prime} \gamma^{\prime 0} \psi_{M}^{\prime} \neq 0
$$

Also, the current

$$
\boldsymbol{J}_{\mathbf{M}}^{5}:=\psi_{\mathbf{M}} \gamma_{3} \tilde{\psi}_{\mathbf{M}}=\left(\overline{\boldsymbol{\psi}}_{M}^{\prime} \gamma^{\prime 5} \gamma^{\prime \mu} \boldsymbol{\psi}_{M}^{\prime}\right) \gamma_{\mu}
$$

is non null as it is easy to verify, and is also lightlike. If the Majorana spinor field was to satisfy the Dirac equation the current $\boldsymbol{J}_{\mathbf{M}}^{5}$ would be also conserved, i.e., $\boldsymbol{\partial}\lrcorner \boldsymbol{J}_{\mathbf{M}}^{5}=0$. In that case we would have a subtle question to answer: how can a massive particle have associated to it currents $\boldsymbol{J}_{\mathbf{M}}$ and $\boldsymbol{J}_{\mathbf{M}}^{5}$ that are ligthlike? What is the meaning of these currents?

Remark 11 Of course, the answer to the above question from the point of view of a first quantized theory is that a Majorana field cannot carry any electric or magnetic charge, i.e., the physical currents $e_{\mathbf{M}} \boldsymbol{J}_{\mathbf{M}}$ and $q_{\mathbf{M}} \boldsymbol{J}_{\mathbf{M}}^{5}$ are null because $e_{\mathbf{M}}=q_{\mathbf{M}}=0$.

\subsection{Making of Majorana Fields that Satisfy the Dirac Equa- tion}

Is it possible to construct a Majorana spinor field that satisfies the Dirac equation?

There are two possibilities of answering yes for the above question.

First possibility: As, e.g., in [18] and [9] we consider ab initio a Majorana field as a quantum field and which is not a dual helicity object. Indeed, define a Majorana quantum field as $\boldsymbol{\psi}_{M}^{\prime}$ as an operator valued field satisfying Majorana condition $\boldsymbol{\psi}_{M}^{\prime c}:=-\gamma^{\prime 2} \overline{\boldsymbol{\psi}}_{M}^{\prime \star}=\boldsymbol{\psi}_{M}^{\prime}$. This condition can be satisfied if we define 19

$$
\boldsymbol{\psi}_{M}^{\prime}(x):=\int \frac{d^{3} \mathbf{p}}{(2 \pi)^{3 / 2}} \sum_{s}\left(u(\mathbf{p}, s) a(\mathbf{p}, s) e^{i p_{\mu} x^{\mu}}+v(\mathbf{p}, s) a^{\dagger}(\mathbf{p}, s) e^{-i p_{\mu} x^{\mu}}\right)
$$

with

$$
\left\{a(\mathbf{p}, s), a^{\dagger}\left(\mathbf{p}^{\prime}, s^{\prime}\right)\right\}=\delta_{s s^{\prime}} \delta\left(\mathbf{p}-\mathbf{p}^{\prime}\right), \quad\left\{a(\mathbf{p}, s), a\left(\mathbf{p}^{\prime}, s^{\prime}\right)\right\}=0
$$

and where the zero momentum spinors $u(\mathbf{0}, s)$ and $v(\mathbf{0}, s)$ are

$$
\begin{aligned}
& u(\mathbf{0}, 1 / 2)=\frac{1}{\sqrt{2}}\left(\begin{array}{l}
1 \\
0 \\
1 \\
0
\end{array}\right), \quad u(\mathbf{0},-1 / 2)=\frac{1}{\sqrt{2}}\left(\begin{array}{l}
0 \\
1 \\
0 \\
1
\end{array}\right), \\
& v(\mathbf{0}, 1 / 2)=\frac{1}{\sqrt{2}}\left(\begin{array}{c}
0 \\
1 \\
0 \\
-1
\end{array}\right), \quad v(\mathbf{0},-1 / 2)=\frac{1}{\sqrt{2}}\left(\begin{array}{c}
-1 \\
0 \\
1 \\
0
\end{array}\right),
\end{aligned}
$$

${ }^{19} \operatorname{Here} \boldsymbol{\psi}_{M}^{\prime \star}:=\int \frac{d^{3} \mathbf{p}}{(2 \pi)^{3 / 2}} \sum_{s}\left(u^{*}(\mathbf{p}, s) a^{\dagger}(\mathbf{p}, s) e^{-i p_{\mu} x^{\mu}}+v^{*}(\mathbf{p}, s) a(\mathbf{p}, s) e^{i p_{\mu} x^{\mu}}\right)$ 
satisfying

$$
\gamma_{0}^{\prime} u(\mathbf{0}, s)=u(\mathbf{0}, s), \quad \gamma_{0}^{\prime} v(\mathbf{0}, s)=-v(\mathbf{0}, s)
$$

Indeed, we can very by explicit calculation that

$$
u(\mathbf{p}, s)=\frac{m+p_{\mu} \gamma^{\prime \mu} \boldsymbol{\gamma}^{\prime 0}}{\sqrt{2 p_{0}\left(p_{0}+m\right)}} u(\mathbf{0}, s), \quad u(\mathbf{p}, s)=\frac{m-p_{\mu} \gamma^{\prime \mu} \boldsymbol{\gamma}^{\prime 0}}{\sqrt{2 p_{0}\left(p_{0}+m\right)}} v(\mathbf{0}, s)
$$

and taking into account Eq.(76) we see that

$$
\left(p_{\mu} \gamma^{\prime \mu}-m\right) u(\mathbf{p}, s)=0, \quad\left(p_{\mu} \gamma^{\prime \mu}+m\right) v(\mathbf{p}, s)=0 .
$$

With this results we can immediately verify that the quantum Majorana field $\boldsymbol{\psi}_{M}^{\prime}(x)$ satisfy the Dirac equation,

$$
\left(i \gamma^{\prime \mu} \partial_{\mu}-m\right) \psi_{M}^{\prime}(x)=0 .
$$

For that Majorana field that is not a dual helicity object we can construct in the canonical way the causal propagator that is nothing more than the standard Feynman propagator for the Dirac equation (see, e.g., [18]).

Remark 12 In a second quantized theory the currents $\boldsymbol{J}_{\mathbf{M}}$ and $\boldsymbol{J}_{\mathbf{M}}^{5}$ are given by the normal product of the field operators and the in this case the current: $\overline{\boldsymbol{\psi}}_{M}^{\prime} \boldsymbol{\gamma}^{\prime 0} \boldsymbol{\psi}_{M}^{\prime}$ : as well known is null, but: $\overline{\boldsymbol{\psi}}_{M}^{\prime} \boldsymbol{\gamma}^{\prime 5} \boldsymbol{\gamma}^{\prime \mu} \boldsymbol{\psi}_{M}^{\prime}$ : is non null. For a proof of these statements, see, e.g., [18] where in particular a consistent quantum field theory for Majorana fields satisfying the Dirac equation is presented, showing in particular that the causal propagator for that field is the standard Feynman propagator of Dirac theory.

Second possibility: In several treatises, e.g., [25, 37] even at the "classical level" it is supposed that any Fermi field must be a Grassmann valued spinor field, i.e., an object where $\phi_{L}^{t}=(\nu \omega)$ and $v, \omega: M \rightarrow \mathcal{G}$, with $\mathcal{G}$ a Grassmann algebra [8, 12, i.e., $v(x)$ and $w(x)$ are Grassmann elements of a Grassmann algebra for all $x \in M$.

In this case it is possible to show that the Majorana field defined, e.g., in 25] by

$$
\Psi^{\prime \mathbf{M}}=\left(\begin{array}{c}
\phi_{L} \\
-\sigma_{2} \phi_{L}^{*}
\end{array}\right)
$$

does satisfy the Dirac equation.

To prove that statement write $\phi_{L}^{t}=\left(\begin{array}{cc}\nu & \omega\end{array}\right)$, where for any $x \in M, v(x)$ and $\omega(x)$ take values in a Grassmann algebra. If $\Psi^{\prime \mathbf{M}}$ does satisfy Dirac equation we must have for $\Psi^{\prime \mathbf{M}}(\mathbf{p})$ at $\mathbf{p}=\mathbf{0}$ that

$$
m\left(\begin{array}{c}
-\boldsymbol{\sigma}_{2} \phi_{L}^{*} \\
\phi_{L}
\end{array}\right)-m\left(\begin{array}{c}
\phi_{L} \\
-\boldsymbol{\sigma}_{2} \phi_{L}^{*}
\end{array}\right)=0
$$

Then we need simultaneously to satisfy the equations

$$
\phi_{L}=-\boldsymbol{\sigma}_{2} \phi_{L}^{*} \text { and } \phi_{L}=\boldsymbol{\sigma}_{2} \phi_{L}^{*}
$$


which at first sight seems to be incompatible, but are not. Indeed, from $\phi_{L}=$ $-\sigma_{2} \phi_{L}^{*}$ we obtain

$$
\nu=i \omega^{*} \text { and } \omega=-i \nu^{*}
$$

and from $\phi_{L}=\sigma_{2} \phi_{L}^{*}$ we obtain

$$
\nu=-i \omega^{*} \text { and } \omega=i \nu^{*} .
$$

So, if we understand the symbol $*$ as denoting the involution defined by Berizin's 20 Eq.(83) is consistent if we take

$$
\nu=\nu^{*} \text { and } \omega=\omega^{*} .
$$

But since for any $c \in \mathbb{C}$ and $\varphi \in \mathcal{G}$ it is $(c \varphi)^{*}=c^{*} \varphi^{*}$ the equation $\nu=i \omega^{*}$ implies

$$
v^{*}=\left(i \omega^{*}\right)^{*}=-i \omega^{* *}=-i \omega
$$

and since $\nu=\nu^{*}$ and $\omega=\omega^{*}$ Eq. (82) implies $\nu=-i \omega^{*}$. Thus, surprisingly as it may be at first sight Eq.(83) is compatible with Eq.(84).

Claim 13 We may then claim that a Majorana field whose components take values in a Grassmann algebra satisfies the Dirac equation. This is consistent with the fact that Dirac equation under these conditions may be derived from a Lagrangian [25, 37. We can also verify that for such a Majorana field the current $J_{\mathbf{M}}=0$.

Remark 14 In resume, from the algebraic point of view there is no difference between elko spinor fields and Majorana spinor fields $\psi_{\mathrm{M}}^{\prime}: M \rightarrow \mathbb{C}^{4}$. However have in mind that the Majorana field defined in 25. (Eq.(80) above) looks like an elko spinor field, but, of course, is not the same object, since the components of an elko spinor fields are for any $x \in M$ complex numbers but the components of $\mathbf{\Psi}^{\prime \mathbf{M}}$ in [25] take values in a Grassmann algebra.

Of course, if we recall that in building a quantum field theory for elkos make automatically the components of elko spinor fields objects taking values in a Grassmann algebra we cannot see any reason for the building of a theory like in 11. Instead we think that elko spinor fields are worth objects of study because they permit the construction of the $\mathcal{K}$ and $\mathcal{M}$ fields introduced above which may describe possible of "magnetic like" particles.

\section{The Causal Propagator for the $\mathcal{K}$ and $\mathcal{M}$ Fields}

We now calculate the causal propagator $\mathcal{S}_{F}\left(x-x^{\prime}\right)$ for, e.g., the $\check{\mathcal{K}} \in \sec \mathcal{C} \ell^{0}(M, \boldsymbol{\eta}) \otimes$ $\mathbb{R}_{1,3}^{0}$ field. Recall from Remark 3 that the $\check{\mathcal{K}}$ field must satisfy

$$
\check{\partial} \check{\mathcal{K}} \boldsymbol{e}_{21}-m \check{\mathcal{K}} \Gamma_{5} \Gamma_{20} \boldsymbol{e}_{0}+\Gamma_{5} q \check{\mathcal{A}} \check{\mathcal{K}}=0 .
$$

\footnotetext{
${ }^{20}$ See pages 66 and of 8
} 
If $\check{\mathcal{K}}_{i}(x)$ is a solution of the homogeneous equation

$$
\check{\partial} \check{\mathcal{K}}_{i} \boldsymbol{e}_{21}-m \check{\mathcal{K}}_{i} \Gamma_{5} \Gamma_{20} \boldsymbol{e}_{0}=0
$$

we can rewrite Eq.(87)as an integral equation

$$
\check{\mathcal{K}}(x)=\check{\mathcal{K}}_{i}(x)+q \int d^{4} y \mathcal{S}_{F}(x, y) \check{\mathcal{A}}(y) \check{\mathcal{K}}(y) \Gamma_{5} \Gamma_{20} \Gamma_{5} .
$$

Putting Eq.(88) in Eq.(87) we see that $\mathcal{S}_{F}(x, y)$ must satisfy for an arbitrary $\check{\mathcal{P}} \in \sec \mathcal{C} \ell(\mathcal{M}, \boldsymbol{\eta}) \otimes \mathbb{R}_{1,3}^{0}$

$$
\check{\partial} \mathcal{S}_{F}(x-y) \check{\mathcal{P}}(y) \boldsymbol{e}_{21}-m \mathcal{S}_{F}(x-y) \check{\mathcal{P}}(y) \boldsymbol{e}_{0}=\delta^{4}(x-y) \check{\mathcal{P}}(y)
$$

whose solution is 17

$$
\mathcal{S}_{F}(x-y) \check{\mathcal{P}}(y)=\frac{1}{(2 \pi)^{4}} \int d^{4} p \frac{\check{p} \check{\mathcal{P}}(y)+m \check{\mathcal{P}}(y) \boldsymbol{e}_{0}}{\check{p}^{2}-m^{2}} e^{-i p_{\mu}\left(x^{\mu}-y^{\mu}\right)} .
$$

For the causal Feynman propagator we get with $E=p_{0}=\sqrt{\mathbf{p}^{2}+m^{2}}$

$$
\begin{aligned}
\mathcal{S}_{F}(x-y) \check{\mathcal{K}}(x) & =\frac{-1}{2(2 \pi)^{3}} \theta\left(t-t^{\prime}\right) \int d^{3} p \frac{\left(\check{p} \check{\mathcal{K}}(y)+m \check{\mathcal{K}}(y) \boldsymbol{e}_{0}\right) \boldsymbol{e}_{21}}{E} e^{-i p_{\mu}\left(x^{\mu}-y^{\mu}\right)} \\
& +\frac{1}{2(2 \pi)^{3}} \theta\left(t-t^{\prime}\right) \int d^{3} p \frac{\left(\check{p} \check{\mathcal{K}}(y)-m \check{\mathcal{K}}(y) \boldsymbol{e}_{0}\right) \boldsymbol{e}_{21}}{E} e^{-i p_{\mu}\left(x^{\mu}-y^{\mu}\right)} .
\end{aligned}
$$

For a scattering problem defining $\check{\mathcal{K}}_{s}=\check{\mathcal{K}}-\check{\mathcal{K}}_{i}$ with $\check{\mathcal{K}}_{i}$ an asymptotic in-state we get when $t \rightarrow \infty$

$$
\check{\mathcal{K}}_{s}(x)=q \int d^{4} y \int d^{3} p \frac{\left(\check{p} \check{\mathcal{A}}(y) \check{\mathcal{K}}(y)+m \check{\mathcal{A}}(y) \check{\mathcal{K}}(y) \boldsymbol{e}_{0}\right) \boldsymbol{e}_{21}}{2 E} e^{-i p_{\mu}\left(x^{\mu}-y^{\mu}\right)}
$$

This permits to define a set of final states $\check{\mathcal{K}}_{f}$ given by

$$
\check{\mathcal{K}}_{f}(x)=q \int d^{4} y \frac{\left(\check{p}_{f} \check{\mathcal{A}}(y) \check{\mathcal{K}}(y)+m \check{\mathcal{A}}(y) \check{\mathcal{K}}(y) \boldsymbol{e}_{0}\right) \boldsymbol{e}_{21}}{2 E_{f}} e^{-i p_{\mu}\left(x^{\mu}-y^{\mu}\right)}
$$

which are plane waves solutions to the free field Dirac-Hestenes equation with momentum $\check{p}_{f}$. Equipped with the $\check{\mathcal{K}}_{i}(x)$ and $\check{\mathcal{K}}_{f}(x)$ we can proceed to calculate the scattering matrix elements, Feynman rules and all that (see details if necessary in [17]).

For the covariant $\boldsymbol{\lambda}$ and $\boldsymbol{\rho}$ fields the causal propagator is the standard Dirac propagator $S_{F}\left(x-x^{\prime}\right)$. Indeed, it can be used to solve, e.g., the csfopde

$$
i \gamma^{\mu} \partial_{\mu} \boldsymbol{\lambda}_{\{-+\}}^{s} \gamma_{21}+m \boldsymbol{\rho}_{\{+-\}}^{a}=0, \quad i \gamma^{\mu} \partial_{\mu} \boldsymbol{\rho}_{\{+-\}}^{a}-m \boldsymbol{\lambda}_{\{-+\}}^{s}=0
$$


once appropriate initial conditions are given. To see this it is only necessary to rewrite the formulas in Eq.94) as

$$
\begin{aligned}
& i \gamma^{\mu} \partial_{\mu} \boldsymbol{\lambda}_{\{-+\}}^{s}-m \boldsymbol{\lambda}_{\{-+\}}^{s}=-m\left(\boldsymbol{\lambda}_{\{-+\}}^{s}+\boldsymbol{\rho}_{\{+-\}}^{a}\right)=\chi, \\
& i \gamma^{\mu} \partial_{\mu} \boldsymbol{\rho}_{\{+-\}}^{a}-m \boldsymbol{\rho}_{\{+-\}}^{a}=m\left(\boldsymbol{\lambda}_{\{-+\}}^{s}+\boldsymbol{\rho}_{\{+-\}}^{a}\right)=\varkappa
\end{aligned}
$$

Eqs.(95) and (96) have solutions

$$
\begin{aligned}
& \lambda_{\{-+\}}^{s}(x)=\int d^{4} y S_{F}(x-y) \chi, \\
& \boldsymbol{\rho}_{\{+-\}}^{a}(x)=\int d^{4} y S_{F}(x-y) \varkappa
\end{aligned}
$$

once we recall that

$$
\left(i \gamma^{\mu} \partial_{\mu}-m\right) S_{F}(x-y)=\delta^{4}(x-y) .
$$

\section{Conclusions}

In [30, 31] it was shown that the massless Dirac-Hestenes equation decouples in a pair of operator Weyl spinor fields, each one carrying opposite magnetic like charges that couple to the electromagnetic potential $A \in \sec \wedge^{1} T^{*} M$ in a non standard way ${ }^{21}$ Here we proposed that the fields $\boldsymbol{\lambda}$ and $\boldsymbol{\rho}$ serves the purpose of building the fields $\mathcal{K}, \mathcal{M} \in \sec \mathcal{C} \ell(M, \eta) \otimes \mathbb{R}_{1,3}^{0}$. These fields are electrically neutral but carry magnetic like charges which permit them to couple to a $\operatorname{spin}_{3,0}$ valued potential $\mathcal{A} \in \sec \bigwedge^{1} T^{*} M \otimes \operatorname{spin}_{3,0}$. If the field $\mathcal{A}$ is of short range the particles described by the $\mathcal{K}$ and $\mathcal{M}$ may interact forming something analogous to dark matter, in the sense that they may form a condensate of spin zero particles with zero total magnetic like charges that do not couple with the electromagnetic field and are thus invisible.

We obtained also the causal propagators for the $\mathcal{K}$ and $\mathcal{M}$ fields, which can be used to calculate scattering matrix elements, Feynman rules, etc 22

Before closing this paper we observe yet that elko spinor fields already appeared in the literature before the publication of [1. A history about these objects may be found in 13, 14. In those papers a Lagrangian equivalent to Eq.(35) written for the covariant spinor fields $\boldsymbol{\lambda}$ and $\boldsymbol{\rho}$ is given. However, the author of those papers did not comment that since the basic csfopde satisfied by the elko spinor fields is by construction the ones given in Eq. (33) and as a consequence these fields, contrary to the claim of [1, must have mass dimension $3 / 2$ and not 1.

We recalled also that as claimed in [1 an elko spinor field (of class five in Lounesto classification) does not satisfy the Dirac equation. According to some

\footnotetext{
${ }^{21}$ In [19] it is proposed that the massless Dirac equation describe ( massless) neutrinos which carry pair of opposite magnetic charges.

${ }^{22}$ At least, we can say that now we have all the ingredients to formulate a quantum field theory for the $\mathcal{K}$ and $\mathcal{M}$ objects if one wish to do so.
} 
claims in the literature (see, e.g., [21] a Majorana spinor field $\psi_{\mathrm{M}}^{\prime}: M \rightarrow \mathbb{C}^{4}$ and which is a dual helicity object (that also belongs to class five in Lounesto classification) does satisfy the Dirac equation. However we showed that this claim is equivocated. At "classical level" a Majorana spinor field can satisfy Dirac equation only if its components for any $x \in M$ take values in a Grassmann algebra.

It is important to emphasize in order to avoid misunderstandings that the theory presented in this paper is an alternative theory to the one originally built in [1] and developed in a series of interesting and challenging papers (see references). It differs drastically from that theory. The main differences are that the equations satisfied by our elko spinor fields of mass dimension $3 / 2$ (see Eq. (33)) and their solutions are trivially Lorentz invariant. In the theory in [1] the elko spinor fields are of mass dimension 1 and that theory breaks Lorentz invariance (see Appendix B). Also our theory gives a prediction of a new type of particle that is electrically and magnetically neutral but has a magnetic like charge which can couple with an $\operatorname{spin}_{3,0}$ valued gauge field. The other theory (for the best of our understanding) does not fix the nature of the field that intermediates the interaction of the particles described by their elko spinor fields of mass dimension 1 . Of course, we do not claim that our theory is better than the other. Which one is appropriate to describe some eventual feature of physical reality (dark matter?) only the future will tell.

Acknowledgement 15 We are grateful to D. V. Ahluwalia for some heated but enlightening discussions on the subject of this paper and to D. Grumiller for a crucial remark. Moreover authors acknowledge in advance any comment they will receive.

\section{A A New Representation of the Parity Opera- tor Acting on Dirac Spinor Fields}

Let $\left\langle\stackrel{\boldsymbol{e}}{\mu}_{\mu}=\frac{\partial}{\partial \mathrm{x}^{\mu}}\right\rangle$ and $\left\langle\boldsymbol{e}_{\mu}=\frac{\partial}{\partial x^{\mu}}\right\rangle$ be two arbitrary orthonormal frames for $T M$ and let $\Sigma_{0}=\left\langle\Gamma^{\mu}=d \mathrm{x}^{\mu}\right\rangle$ and $\Sigma=\left\langle\gamma^{\mu}=d x^{\mu}\right\rangle$ be the respective dual frames. Of course, $\stackrel{\boldsymbol{e}}{0}_{0}$ and $\boldsymbol{e}_{0}$ are inertial reference frames [31] and we suppose now that $\boldsymbol{e}_{0}$ is moving relative to $\stackrel{\boldsymbol{e}}{0}_{0}$ with 3 -velocity $\mathbf{v}=\left(\mathbf{v}^{1}, v^{2}, v^{3}\right)$, i.e.,

$$
\boldsymbol{e}_{0}=\frac{1}{\sqrt{1-v^{2}}} \stackrel{\circ}{0}_{0}-\sum_{i=1}^{3} \frac{v^{i}}{\sqrt{1-v^{2}}} \stackrel{\circ}{i}_{i}
$$

Let $\Xi_{u_{0}}$ and $\Xi_{u}$ be the spinorial frames associated with $\Sigma_{0}$ and $\Sigma$. Consider a Dirac particle at rest in the inertial frame $\check{\boldsymbol{e}}_{0}$ (take as a fiducial frame). The triplet $\left(\psi_{0}, \Sigma_{0}, \Xi_{0}\right)$ is the representative of the wave function of our particle in $\left(\Sigma_{0}, \Xi_{0}\right)$ and of course, its representative in $(\Sigma, \Xi)$ is $(\psi, \Sigma, \Xi)$. Now,

$$
\psi=u \psi_{0}
$$


where $u$ describes in the spinor space the boost sending $\Gamma^{\mu}$ to $\gamma^{\mu}$, i.e., $\gamma^{\mu}=u$ $\Gamma^{\mu} u^{-1}=\Lambda_{\nu}^{\mu} \Gamma^{\nu}$. Now, the representative of the parity operator in $\left(\Sigma_{0}, \Xi_{0}\right)$ is $\mathcal{P}_{u_{0}}$ and in $(\Sigma, \Xi)$ is $\mathcal{P}_{u}$; We have according to our dictionary (Eq.(99) that

$$
\mathcal{P}_{u} \psi=\gamma^{0} \psi \gamma^{0}, \quad \mathcal{P}_{u_{0}} \psi_{0}=\Gamma^{0} \psi_{0} \Gamma^{0},
$$

or

$$
\mathcal{P}_{u} \boldsymbol{\Psi}=\gamma^{0} \mathcal{R} \boldsymbol{\Psi}, \quad \mathcal{P}_{u_{0}} \boldsymbol{\Psi}_{0}=\Gamma^{0} \mathcal{R} \boldsymbol{\Psi}_{0},
$$

where $\psi$ and $\psi_{0}$ are Dirac ideal real spinor field 23

$$
\boldsymbol{\Psi}=\psi \frac{1}{2}\left(1+\gamma^{0}\right), \quad \boldsymbol{\Psi}_{0}=\psi_{0} \frac{1}{2}\left(1+\Gamma^{0}\right),
$$

and if the momentum of our particle is the covector field $\boldsymbol{p}=\stackrel{\circ}{p}_{\mu} \Gamma^{\mu}=p_{\mu} \gamma^{\mu}$ with $\left(\stackrel{\circ}{0}_{0}, \stackrel{\circ}{p}_{1}, \stackrel{\circ}{p}_{2}, \stackrel{\circ}{p}_{3}\right):=(m, \mathbf{0})$ and $\left(p_{0}, p_{1}, p \stackrel{\circ}{p}_{2}, p_{3}\right):=(E, \mathbf{p})$ (and of course $p_{\mu}=$ $\left.\Lambda_{\mu}^{\nu} \stackrel{\circ}{p}_{\mu}=\Lambda_{\mu}^{0} \dot{p}_{0}\right) \mathcal{R}$ an the operator such that if $\psi=\phi(\mathbf{p}) e^{i \boldsymbol{p} \boldsymbol{x}}$ then

$$
\mathcal{R} \psi=\phi(\mathbf{p}) e^{-i \boldsymbol{p}_{\mu} \boldsymbol{x}^{\mu}}=\phi(\mathbf{p}) e^{-i\left(\boldsymbol{p}_{0} \boldsymbol{x}^{0}-p_{i} x^{i}\right)} .
$$

Also $u \mathcal{R}=\mathcal{R} u$ and clearly $\mathcal{R} \psi_{0}=\psi_{0}$. Now,

$$
u \mathcal{P}_{u_{0}} u^{-1} u \boldsymbol{\Psi}_{0}=u \Gamma^{0} \mathcal{R} \boldsymbol{\Psi}_{0}=u \Gamma^{0} u^{-1} \mathcal{R} u \boldsymbol{\Psi}_{0}=\gamma^{0} \mathcal{R} \boldsymbol{\Psi},
$$

from where it follows that

$$
\mathcal{P}_{u}=u \mathcal{P}_{u_{0}} u^{-1} .
$$

Now we rewrite $\mathcal{P}_{u} \boldsymbol{\Psi}=\gamma^{0} \mathcal{R} \boldsymbol{\Psi}$ as

$$
\begin{aligned}
\mathcal{P}_{u} \boldsymbol{\Psi} & =\frac{\stackrel{\triangleright}{0}_{0}}{m} u \Gamma^{0} \mathcal{R} \boldsymbol{\Psi}_{0}=\frac{\stackrel{p}{0}_{0}}{m} u \Gamma^{0} u^{-1} u \mathbf{\Psi}_{0}, \\
& =\frac{\check{p}_{0}}{m} \Lambda_{\mu}^{0} \gamma^{\mu} \boldsymbol{\Psi}=\frac{1}{m} p_{\mu} \gamma^{\mu} \boldsymbol{\Psi} .
\end{aligned}
$$

We conclude that the parity operator in an arbitrary orthonormal and spin frames $(\Sigma, \Xi)$ acting on a Dirac ideal spinor field $\psi)$ is

$$
\mathcal{P}=\mathcal{P}_{u}=\frac{1}{m} p_{\mu} \gamma^{\mu}
$$

Of course, when applied to covariant spinor fields $\psi: M \rightarrow \mathbb{C}^{4}$ the operator $\mathcal{P}$ is represented by

$$
\boldsymbol{P}=\frac{1}{m} p_{\mu} \gamma^{\mu} .
$$

A derivation of this result using covariant spinor fields (and which can be easy generalized for arbitrary higher spin fields) has been obtained in [36.

${ }^{23}$ See 31 for details. 


\section{B Correct Value for the Fourier Transform of $\mathcal{G}(\mathbf{p})$}

According to the theory of elko spinor fields as originally developed in [1] (see also $2,3,4,4,6,6]$, the evaluation of the anticommutator of an elko spinor field with its canonical momentum gives

$$
\left\{\Lambda(\mathbf{x}, t), \Pi(\mathbf{x}, t\}=i \delta\left(\mathbf{x}-\mathbf{x}^{\prime}\right) \mathbb{I}+i \int \frac{d^{3} p}{(2 \pi)^{3}} e^{i \mathbf{p} \cdot\left(\mathbf{x}-\mathbf{x}^{\prime}\right)} \mathcal{G}(\mathbf{p}),\right.
$$

with

$$
\mathcal{G}(\mathbf{p}):=\gamma^{5} \gamma^{\mu} n_{\mu}(\mathbf{p})
$$

where the spacelike $\mathbf{p}$-dependent field $n=n_{\mu}(\mathbf{p}) \mathbf{e}_{\mu}$ is

$$
\begin{gathered}
\left(n_{0}(\mathbf{p}), n_{1}(\mathbf{p}), n_{2}(\mathbf{p}), n_{3}(\mathbf{p})\right):=(0, \mathbf{n}(\mathbf{p})), \\
\mathbf{p}=(p \cos \theta, p \sin \theta \cos \varphi, p \sin \theta \sin \varphi) \\
\mathbf{n}(\mathbf{p}):=\frac{1}{\sin \theta} \frac{\partial}{\partial \varphi}\left(\frac{\mathbf{p}}{|\mathbf{p}|}\right)=(-\sin \varphi, \cos \varphi, 0) \\
=\left(-\tau\left(1+\tau^{2}\right)^{-1 / 2}, \tau\left(1+\tau^{2}\right)^{-1 / 2}, 0\right), \quad \tau=p_{y} / p_{x} .
\end{gathered}
$$

Putting $\boldsymbol{\Delta}=\mathbf{x}-\mathbf{x}^{\prime}$ it i: 24

$$
\begin{aligned}
\hat{\mathcal{G}}(\boldsymbol{\Delta}) & =\int \frac{d^{3} p}{(2 \pi)^{3}} e^{i \mathbf{p} \cdot\left(\mathbf{x}-\mathbf{x}^{\prime}\right)} \mathcal{G}(\mathbf{p}) \\
& =-\gamma^{5} \gamma^{1} P(\boldsymbol{\Delta})+\gamma^{5} \gamma^{2} Q(\boldsymbol{\Delta})
\end{aligned}
$$

with

$$
P(\boldsymbol{\Delta})=-\frac{i}{2 \pi} \delta\left(\Delta_{z}\right) \frac{\Delta_{y}}{\left(\Delta_{x}^{2}+\Delta_{y}^{2}\right)^{\frac{3}{2}}}, \quad Q(\boldsymbol{\Delta})=\frac{i}{2 \pi} \delta\left(\Delta_{z}\right) \frac{\Delta_{x}}{\left(\Delta_{x}^{2}+\Delta_{y}^{2}\right)^{\frac{3}{2}}}
$$

Remark 16 In [?] the integral in Eq.(115) has been evaluated for the case when $\boldsymbol{\Delta}$ lies in the direction of one of the spatial axes $\mathbf{e}_{i}=\partial / \partial x^{i}$ of an arbitrary inertial reference frame $\mathbf{e}_{0}=\partial / \partial x^{0}$ (where $\left(x^{0}, x^{1}, x^{2}, x^{3}\right)$ are coordinates in Einstein-Lorentz-Poincaré gauge naturally adapted to $\mathbf{e}_{0}$ [31, 33]]). We note that the evaluation of each one of the integrals in [?] is correct, but they do not express the values of the Fourier transform $\hat{\mathcal{G}}\left(\mathbf{x}-\mathbf{x}^{\prime}\right)$ for the particular values of $\boldsymbol{\Delta}$ used in the calculations of those integrals. It is not licit to fix a priori two of the components of $\boldsymbol{\Delta}$ as being null to calculate the integral $(2 \pi)^{-3} \int d^{3} p e^{i \mathbf{p} \cdot\left(\mathbf{x}-\mathbf{x}^{\prime}\right)} \mathcal{G}(\mathbf{p})$ for this procedure excludes the singular behavior in the sense of distributions of the Fourier integral. So, it is wrong the statement in [10] that elko theory as constructed originally in [1] is loca 25.

\footnotetext{
${ }^{24}$ This result has been also found by Ahluwalia and Grumiller. We find the above result without knowing their calculations, which Ahluwalia informed us will be reported in a forthcoming paper.

${ }^{25}$ An errata has been sent to PRD.
} 


\section{B.1 Plane of Nonlocality and Breakdown of Lorentz In- variance}

When $\Delta_{z} \neq 0, \hat{\mathcal{G}}\left(\mathbf{x}-\mathbf{x}^{\prime}\right)$ is null the anticommutator is local and thus there exists in the elko theory as constructed in [1, 5] an infinity number of "locality directions". On the other hand $\hat{\mathcal{G}}\left(\mathbf{x}-\mathbf{x}^{\prime}\right)$ is a distribution with support in $\Delta_{z}=0$. So,the directions $\boldsymbol{\Delta}=\left(\Delta_{x}, \Delta_{y}, 0\right)$ are nonlocal in each arbitrary inertial reference frame $\mathbf{e}_{0}$ chosen to evaluate $\hat{\mathcal{G}}\left(\mathbf{x}-\mathbf{x}^{\prime}\right)$. Recall that given an inertial (coordinate) reference frame frame $\mathbf{e}_{0}=\partial / \partial x^{0}$ in Minkowski spacetime there exists [11] and infinity of triples of vector fields $\left\{\mathbf{e}_{1}^{(k)}=\partial / \partial x_{(k)}^{1}, \mathbf{e}_{1}^{(k)}=\partial / \partial x_{(k)}^{2}, \mathbf{e}_{1}^{(k)}=\right.$ $\left.\partial / \partial x_{(k)}^{3}\right\}$ (with $\left(x^{0}, x_{(k)}^{1}, x_{(k)}^{2}, x_{(k)}^{3}\right)$, coordinates in Einstein-Lorentz-Poincaré gauge [31] naturally adapted to $\mathbf{e}_{0}$ differing by a spatial rotation) which constitutes a global section of the frame bundle. So, the labels $x, y$ and $z$ directions in inertial reference frame $\mathbf{e}_{0}$ are arbitrary (a mere convention) and thus without any physical significance. This means that the theory as constructed in [1] breaks in each inertial reference frame rotational invariance and since in different inertial references frames there are different $(x, y)$ planes the theory breaks also Lorentz invariance. This means that the theory as constructed in [1 breaks in each inertial reference frame rotational invariance and since in different inertial references frames there are different ( and moreover arbitrary) $(x, y)$ planes the theory breaks also Lorentz invariance. This odd feature (according to our view) of the theory of elko spinor fields as constructed originally in [1] was eventually the main reason that lead us to the investigation described in this paper. However if this odd effect will be observed in experiments we must agree that the original version of elko theory is indeed a science breakthrough. The one who lives will know.

\section{References}

[1] Ahluwalia-Khalilova, D. V. and Grumiller, D., Spin-half Fermions with Mass Dimension One: Theory, Phenomenology, and Dark Matter, JCAP 07012 (2005) [hep-th/0412080]

[2] Ahluwalia-Khalilova, D. V. and Grumiller, D., Dark Matter: A Spin One Half Fermion Field with Mass Dimension One?, Phys.Rev. D 72067701 (2005) [hep-th/0410192]

[3] Ahluwalia, D. V, Review of Quantum Field Theory, Second Edition. By Lewis H. Ryder (Cambridge University Press, United Kingdom, 1996), Found. Phys. 28, 527-528 (1998).

[4] Ahluwalia, D. V., and Horvath, S. P., Very Special Relativity of Dark Matter; The elko Connection, JHEP 11, 078 (2010). [arXiv: 1008.0436v2 [hep-ph] ] 
[5] Ahluwalia, D. V., Lee, C. Y., and Scritt, D, Elko as Self Interacting Fermionic Dark Matter with Axis of Locality, Phys. Lett. B. 687, 248-252 (2010).

[6] Ahluwalia, D. V., Lee, C. Y., and Scritt, D., Self-Interacting Matter With an Axis of Locality, Phys. Rev. D 83, 065017 (2011).

[7] Berestetskiŭ, V. B., Lifishitz, E. M., and Pitaesvskiǔ, L., Relativistic Quantum Theory, Part I, Pergamon Press, New York, 1971.

[8] Berezin, F. A., The Method of Second Quantization, Academic Press, New York, 1966.

[9] Cahill, P. and Cahill, K., Learning about Spin-One-Half Fields, Eur. J. Phys. 27, 29-47 (2006) and corrigenda at Eur. J. Phys. 28, 145 (2006).

[10] Capelas de Oliveira, E., and Rodrigues, W. A. Jr., Comment on "Selfinteracting Elko Dark Matter with an Axis of Locality" Phys. Rev. D 86, 128501 (2012).

[11] Choquet-Bruhat, Y., DeWitt-Morette, C., Dillard-Bleick, M., Analysis, Manifolds and Physics (revised edition), North Holland, Amsterdam, 1982.

[12] DeWitt, B., Supermanifold, Cambridge University Press, Cambridge, 1984.

[13] Dvoeglazov, V.V., Majorana Neutrino: Chirality and Helicity, J. Phys. Conf. Ser. 343, 012033 (2012). [arXiv:1108.4991v2 [math-ph]]

[14] Dvoeglazov, V.V., How to construct Self/Anti-Self Charge Conjugate States for Higher Spins? [arXiv:1210.4401v1 [math-ph]]

[15] Gradshteyn, I. S. and Ryzhik, I. M., Table of Integrals Series and Products (fourth edition), 1965. Academic Press, New York,

[16] Greiner, W., Relativistic Quantum Mechanics, pp.84-85, Springer-Verlag, Berlin,1994.

[17] Gull, S., Doran, C, and Lasenby, A., Electron Physics II, in Baylis, W. E. (ed.), Clifford (Geometrical) Algebras, pp.129-145, Birkhaüser, Boston, 1996.

[18] Leite Lopes, J., Lectures on Symmetries, Gordon and Breach, New York, 1969.

[19] Lochak, G., Wave Equation for a Magnetic Monopole, Int. J. Theor. Phys. 24, 1019-1050 (1985).

[20] Lounesto, P., Clifford Algebras and Spinors, Cambridge University Press, Cambridge, 1997.

[21] Maggiore, M., A Modern Introduction to Quantum Field Theory, Oxford Univ. Press, Oxford, 2005. 
[22] Marshak, R. E. and Sudarshan, E.C.G., Introduction to Elementary Particle Physics, John Wiley, New York 1961.

[23] Mosna, R. A., and Rodrigues, W. A. Jr., The Bundles of Algebraic and Dirac-Hestenes Spinor Fields, J. Math. Phys. 45, 2945-2966 (2004). [arXiv:math-ph/0212033]

[24] Pal, P. B., Dirac, Majorana and Weyl Fermions, Am. J. Phys. 79, 485-498 (2011). [arXiv:1006.1718v2 [hep-ph]]

[25] Ramond, P., Field Theory: A Modern Primer (second edition), AddisonWesly Publ. Co., Reading, MA, 1989.

[26] Rodrigues, W. A. Jr., Algebraic and Dirac-Hestenes Spinors and Spinor Fields, J. Math. Phys. 45, 2908-2994 (2004). [arXiv:math-ph/0212030]

[27] da Rocha, R. and Rodrigues, W. A. Jr., Where are elko Spinor Fields in Lounesto Spinor Field Classification?, Mod. Phys. Lett. A 21, 65-74 (2006) [math-ph/0506075]

[28] da Rocha, R. and da Silva,J. M. Hoff, From Dirac Spinor Fields to elko, J. Math. Phys.48 123517 (2007). [arXiv:0711.1103 [math-ph]].

[29] da Rocha, R., and da Silva,J. M. Hoff, elko, Flagpole and Flag-Dipole Spinor Fields, and the Instanton Hopf Fibration, Adv. Appl. Clifford Algebras 20, 847-870 (2010). [arXiv:0811.2717 [math-ph]]

[30] Rodrigues, W. A. Jr., The Relation Between Maxwell, Dirac and the Seiberg-Witten Equations, Int. J. Math. and Math. Sci. 2003, 2707-2734 (2003). [arXiv:math-ph/0212034]

[31] Rodrigues, W. A. Jr., and Capelas de Oliveira, E., The Many Faces of Maxwell, Dirac and Einstein Equations. A Clifford Bundle Approach. Lecture Notes in Physics 722, Springer, Heidelberg, 2007.

[32] Ryder, L. H., Quantum Field Theory (second edition), Cambridge Univ. Press, Cambridge, 1996.

[33] Sachs, R. K., and Wu, H., General Relativity for Mathematicians, Springer, New York, 1977.

[34] da Silva, J..M. Hoff, and da Rocha, R., From Dirac Action to elko Action, Int. J. Mod. Phys. A 24, 3227-3242 (2009). [arXiv:0903.2815 [math-ph] ]

[35] Schweber, S. S., An Introduction to Relativistic Quantum Field Theory, Harper and Row, New York, 1964.

[36] Sperança, L. D., An Identification of the Dirac Operator with the Parity Operator [arXiv:1304.4794 [math-ph]] 
[37] Ticciati, R., Quantum Theory for Mathematicians, Cambridge University Press, Cambridge, 1999.

[38] Yang, C. N., and Tiomno, J., Reflection Properties of Spin 1/2 Fields and a Universal Fermi-Type Interaction, Phys. Rev. 88, 495-498 (1950). 